ARTICLE

\title{
Direct measurements of interfacial adhesion in 2D materials and van der Waals heterostructures in ambient air
}

\author{
Hossein Rokni ${ }^{1} \&$ Wei Lu (iD ${ }^{1,2}$
}

Interfacial adhesion energy is a fundamental property of two-dimensional (2D) layered materials and van der Waals heterostructures due to their intrinsic ultrahigh surface to volume ratio, making adhesion forces very strong in many processes related to fabrication, integration and performance of devices incorporating 2D crystals. However, direct quantitative characterization of adhesion behavior of fresh and aged homo/heterointerfaces at nanoscale has remained elusive. Here, we use an atomic force microscopy technique to report precise adhesion measurements in ambient air through well-defined interactions of tip-attached 2D crystal nanomesas with $2 \mathrm{D}$ crystal and $\mathrm{SiO}_{x}$ substrates. We quantify how different levels of short-range dispersive and long-range electrostatic interactions respond to airborne contaminants and humidity upon thermal annealing. We show that a simple but very effective precooling treatment can protect 2D crystal substrates against the airborne contaminants and thus boost the adhesion level at the interface of similar and dissimilar van der Waals heterostructures. Our combined experimental and computational analysis also reveals a distinctive interfacial behavior in transition metal dichalcogenides and graphite $/ \mathrm{SiO}_{x}$ heterostructures beyond the widely accepted van der Waals interaction.

\footnotetext{
${ }^{1}$ Department of Mechanical Engineering, University of Michigan, Ann Arbor, MI 48109, USA. ${ }^{2}$ Department of Materials Science and Engineering, University

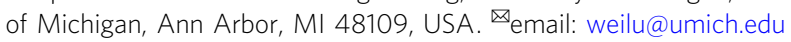


T wo-dimensional layered materials (2DLMs), such as graphene, hexagonal boron nitride ( $\mathrm{hBN}$ ), transition metal dichalcogenides (TMDs: e.g., $\mathrm{MoS}_{2}$ and $\mathrm{WS}_{2}$ ), and many others, with strong in-plane covalent bonding and weak out-ofplane van der Waals (vdW) interactions exhibit a unique combination of high elasticity, high mechanical flexibility, visual transparency, and superior (opto)electronic performance, making them ideally suited to modern devices, such as photovoltaic devices, hybrid electrochemical capacitors, lithium- and sodiumion batteries, hydrogen evolution catalysis, transistors, photodetectors, DNA detection, and memory devices ${ }^{1}$. However, intrinsic ultrahigh surface-to-volume ratio in 2DLMs requires intimate knowledge of interfacial adhesion between two adjacent layers of similar or dissimilar 2DLMs and also between 2DLMs and their supporting substrate. In particular, such a fundamental mechanical property plays a central role not only in synthesis, transfer, and manipulation of 2DLMs but also in fabrication, integration, and performance of 2DLM-incorporated devices.

In general, fabrication of $2 \mathrm{D}$ systems involves transferring 2DLMs from their growth substrate or bulk stamp onto a target substrate using different transfer-printing techniques. A better understanding of the adhesion energy between 2DLMs and the various substrates involved is highly desired as an essential step toward enhancing the transfer efficiency and thickness uniformity of printed flakes and thus producing high-quality, large-scale 2D electronic device arrays at microscale and nanoscale ${ }^{2}$. The interfacial adhesion between 2DLMs and their neighbors is also an important parameter for the mechanical integrity of the device whose operation is highly influenced by slippage and delamination of 2DLMs during thermal and mechanical loadings. As such, a 2DLM needs to make secure contact not only with supporting substrates and metallic interconnects in 2DLM-based devices but also with other 2DLMs and encapsulation layers in vdW heterostructure devices 3,4 .

Newly emerged vdW heterostructures-stacks of individual monolayer flakes of different 2DLMs assembled layer by layeroffer a variety of new physical properties, thanks to the full spectrum of electronic properties in 2DLMs, from conducting graphene to semiconducting TMDs, to insulating hBN. An essential feature of such heterostructures is atomically clean interfaces to achieve the best device performance-any interfacial contamination (e.g., blisters) results in deterioration of transport properties $^{5}$. As such, wet transfer and dry pick-and-lift transfer techniques are widely used for assembly of vdW heterostructures. However, both direct mechanical assembly techniques rely strongly on vdW interactions between the $2 \mathrm{D}$ crystals, and as a result, an accurate quantification of interfacial adhesion between different 2DLMs is crucial for the mass production of blister-free $\mathrm{vdW}$ hetersostructures.

Fascinating interlayer vdW-dependent properties of similar and dissimilar 2DLMs provide a unique opportunity to study the nature of electronic structure and band alignment, interfacial thermal and electrical resistance, ion intercalation and deintercalation process, interfacial nanofluidic transport and drug delivery behavior, photon absorption and photocurrent/photovoltaic production, interfacial charge polarization and redistribution, spin-orbit coupling, and many others in layered material-based devices ${ }^{6-10}$. Notably, interfacial electrical, mechanical, optoelectronic, magnetic, and thermal properties of layered materials can also interact in a rather complex way. For instance, formation of any delamination-motivated surface corrugations in 2DLMs can give rise to local strain distribution and curvature-induced rehybridization, which modify the electronic structure and local charge distribution; create polarized carrier puddles and dipole moment; induce pseudomagnetic fields; and thus alter magnetic, optical, and electrical properties as well as chemical surface reactivity ${ }^{11}$. Moreover, the vdW interaction as a key medium for the stress transfer both within and across the interface of 2DLMs can highly impact their thermal and electrical properties in such a way that a $2 \mathrm{D}$ layered system can act as a heat conductor or insulator and/or a semimetal or electrical insulator through strain engineering ${ }^{12-14}$.

The interfacial physical and chemical behavior of layered materials becomes even more complicated when we consider that airborne contaminants are an inevitable part of any vdW heterostructures, and therefore addressing quantitatively to what degree their interfacial adhesion energy (IAE) is influenced by interfacial contaminants and nanoblisters and how to effectively remove them is of fundamental and technological importance for the continued development of such promising materials. However, many attempts have been made over the past six decades to measure the IAE of 2D crystals either in high vacuum or under a contamination-free environment. Among them, few direct IAE measurements of 2D crystals have been reported with a particular focus on graphite $(\mathrm{G})$ crystal $^{15-21}$. For instance, the IAE at the intact $G / G$ homointerface was reported using micro-force sensing probe measurements on $4-\mu \mathrm{m}$-wide square mesas $(0.37 \pm$ $0.01 \mathrm{~J} \mathrm{~m}^{-2}$, ref. ${ }^{15}$ ) and atomic force microscopy (AFM)-assisted shearing measurements on $3-\mu \mathrm{m}$-wide square mesas $\left(0.35 \mathrm{~J} \mathrm{~m}^{-2}\right.$, ref. $\left.{ }^{16}\right)$ and circular mesas of $100-600 \mathrm{~nm}$ in diameter $(0.227 \pm$ $0.005 \mathrm{~J} \mathrm{~m}^{-2}$, ref. $\left.{ }^{17}\right)$. Moreover, there is only one measured IAE value of $0.22 \mathrm{~J} \mathrm{~m}^{-2}$ at the $\mathrm{MoS}_{2} / \mathrm{MoS}_{2}$ homointerface using a nanomechanical cleavage technique ${ }^{18}$, whereas, to the best of our knowledge, no IAE measurement at the $\mathrm{hBN} / \mathrm{hBN}$ homointerface yet exists. Also, the vdW interaction at $\mathrm{G} / \mathrm{hBN}$ and $\mathrm{G} / \mathrm{MoS}_{2}$ heterointerfaces was studied using a G-wrapped sharp tip with an unknown contact area, allowing the measurements of critical adhesion forces between $\mathrm{G} / \mathrm{G}, \mathrm{G} / \mathrm{hBN}$, and $\mathrm{G} / \mathrm{MoS}_{2}$ in high vacuum at room temperature ${ }^{19}$. Although a considerable number of experimental and theoretical methods have been proposed to study the IAE of 2D crystals in general and G crystal specifically, there is significant diversity in the reported IAE values, where the exact cause of the variation in their IAE values has still remained to be elucidated by a comprehensive and accurate experimental technique.

In this work, the first direct nanoscale quantification of IAE at both fresh and aged 2D vdW homointerfaces/heterointerfaces is performed under different annealing temperatures. To this end, force-displacement $(F-d)$ curves with piconewton-subnanometer resolution are recorded upon retraction of AFM tip-attached 2D crystal nanomesas from tens-of-nm-thick fresh and aged 2D crystal substrates and bare $\mathrm{SiO}_{x} / \mathrm{Si}$ substrate under controlled ambient conditions in the near equilibrium regime (Fig. 1a). The annealing temperature of nanocontact interfaces is precisely controlled in the range of $-15-300^{\circ} \mathrm{C}$ by a microheater on the top (left inset of Fig. 1a) and a cooling stage underneath the $\mathrm{SiO}_{x} /$ Si substrate. Aged substrates are prepared by two different aging conditions where the freshly exfoliated $2 \mathrm{D}$ crystal and bare $\mathrm{SiO}_{x} /$ Si substrates are either exposed to the ambient air directly (hereafter referred to simply as untreated substrates) or kept at subzero temperature, followed by the air exposure (referred to as precooling-treated substrates). Among many different combinations of dissimilar 2DLMs, we focus on the interlayer vdW behavior of conducting $\mathrm{G}$, insulating $\mathrm{hBN}$, and semiconducting $\mathrm{MoS}_{2}$ crystals as a model system for a large class of $2 \mathrm{D} \mathrm{vdW}$ heterostructure systems. Since direct nanoscale probing of weak $\mathrm{vdW}$ interactions in 2DLMs and vdW heterostructures requires a unique combination of high-resolution imaging, precise mechanical manipulation, and accurate in situ interfacial adhesion measurements, nano-sized square (circular) 2D crystal mesas with a width (diameter) of 55-65 nm are attached to an in situ flattened AFM tip, which is precoated with an ultrathin adhesive 


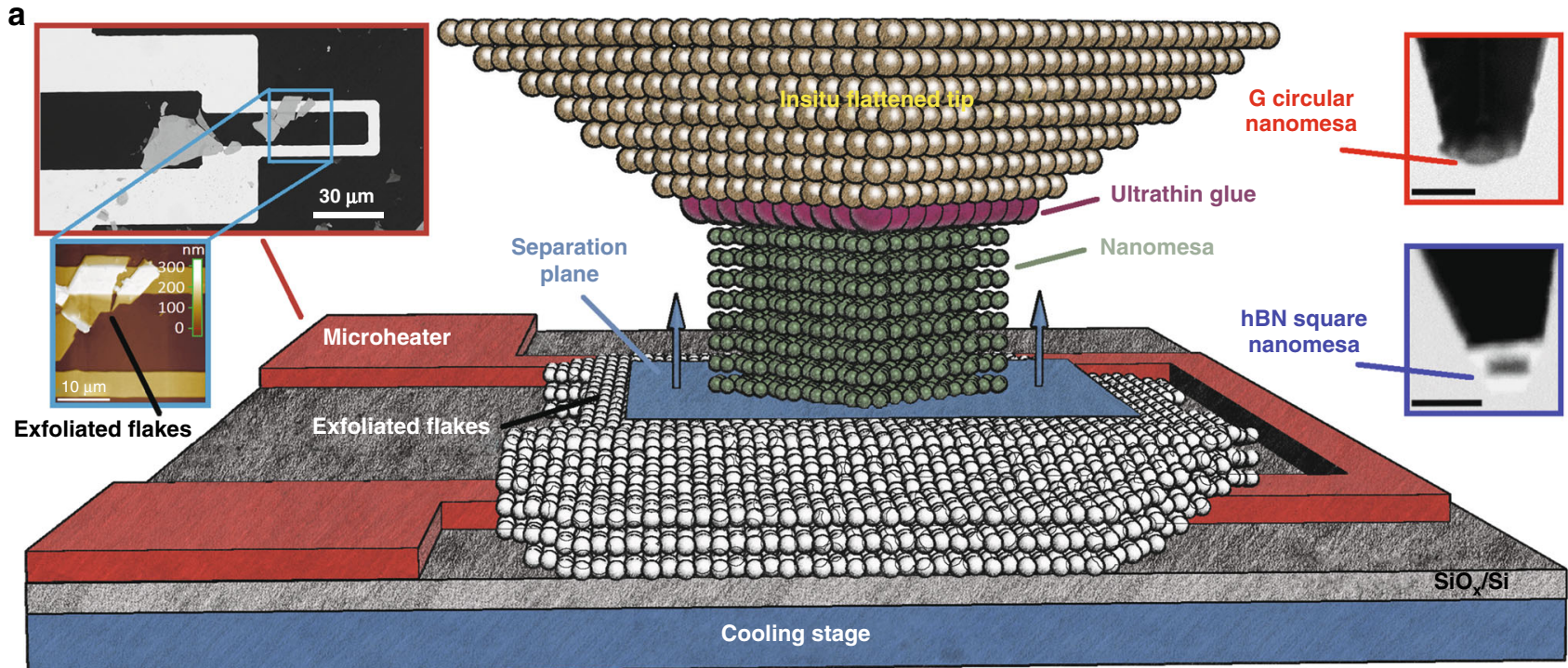

b

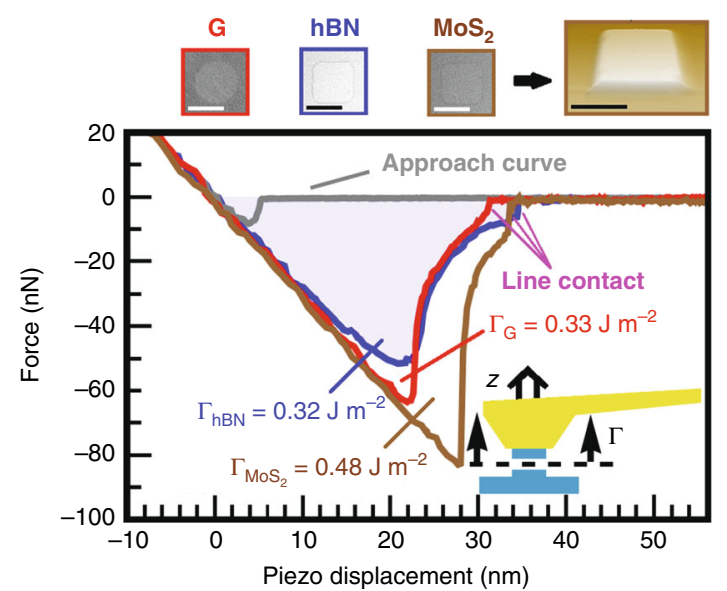

d

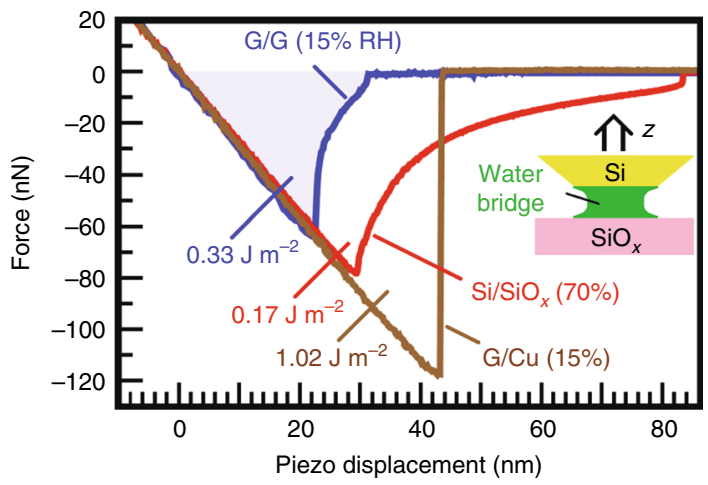

polymer at the apex (right inset of Fig. 1a and Supplementary Note 1). Using nano-sized 2D crystal tips with a very well-defined geometric shape parallel to the substrate together with accurate determination of spring constant of the probe is an essential prerequisite for the detailed characterization of nanoscale vdW interfaces and the accurate extraction of interfacial adhesion properties of 2DLMs (Supplementary Note 2).
C
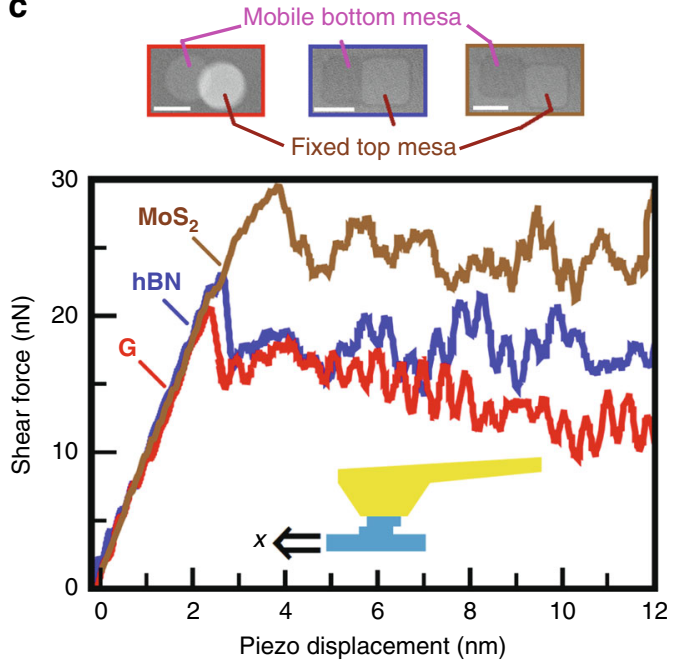

e

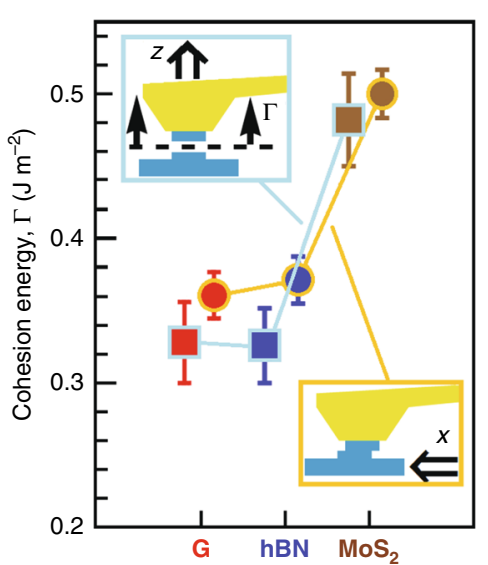

Results

Cohesion energy in 2D crystals. During the attachment of nanomesas to the glue-coated tip, $F-d$ curves can be recorded as the tip is gently pulled away from the substrate surface in a direction perpendicular to the single basal plane of $2 \mathrm{D}$ crystal, leading to pulling off the upper section of the nanomesa (attached to the tip apex) from the lower section (fixed to the $2 \mathrm{D}$ crystal 
Fig. 1 AFM-assisted experimental set-up and cohesion energy measurements. a Schematic of the AFM set-up used to perform interfacial adhesion measurements under different annealing temperatures. Left inset: SEM image of the microheater with an $\mathrm{MoS}_{2}$ flake exfoliated on the heating line whose corresponding AFM image was taken by the G crystal tip. Right Inset: SEM images of the tip-attached G circular nanomesa (top image) and hBN square nanomesa (bottom image). Scale bars indicate $100 \mathrm{~nm}$. b Typical retraction $F$ - $d$ curves recorded at the intact homointerface of $\mathrm{G}$ (in red), hBN (in blue), and $\mathrm{MoS}_{2}$ (in brown) crystals and also the approach $\mathrm{F}$ - $d$ curve (in gray) recorded at the hBN tip/substrate homointerface. The light blue-shaded area under the retraction curve at the hBN homointerface represents the cohesion energy in units of Joules. Each raw data set was given an offset to provide the same equilibrium position for all F-d curves. Top panel: SEM images of lower section of the nanomesas on their corresponding bulk substrate after the full tip retraction. Perspective AFM image corresponding to the SEM image of the $\mathrm{MoS}_{2}$ nanomesa is also shown. Scale bars indicate $50 \mathrm{~nm}$. c Typical shear force-lateral piezo displacement curves recorded at the intact homointerface of G (in red), hBN (in blue), and $\mathrm{MoS}_{2}$ (in brown) crystals. Schematic inset shows that the $2 \mathrm{D}$ crystal substrate moves along the long axis of the cantilever tip at zero contact force. Top panel: Corresponding SEM images of the sheared G, hBN, and $\mathrm{MoS}_{2}$ nanomesas. Scale bars indicate $50 \mathrm{~nm}$. d Typical retraction F- $d$ curves recorded at the interface of tip-attached $\mathrm{G}$ nanomesa/G substrate (in blue), bare $\mathrm{Si}$ tip-attached water nanomeniscus $/ \mathrm{SiO}_{x}$ substrate (in blue), and tip-attached $\mathrm{G}$ mesa/Cu substrate (in brown) at the relative humidity of 15,70 , and $15 \%$, respectively. Schematic inset shows the meniscus rupture between the in situ flattened Si tip and the $\mathrm{SiO} \times x / \mathrm{Si}$ substrate, where the energy required to rupture the nanomeniscus is roughly obtained by dividing the area under the $F-d$ curve over the area of the flat tip. e Cohesion energy of $\mathrm{G}, \mathrm{hBN}$, and $\mathrm{MoS}_{2}$ crystals obtained by normal force measurements (squares with cyan borders) and shear force measurements (circles with orange borders) at room temperature. Data are presented as average \pm standard deviation.

substrate). Similarly, the shear $F-d$ curves are recorded as the nanomesa is sheared along the long axis of the cantilever tip rather than perpendicular to its long axis to obtain more accurate shear force measurements. Figure $1 \mathrm{~b}$, $\mathrm{c}$ illustrate typical retraction $F-d$ and shear $F-d$ curves, respectively, at the intact $\mathrm{G}, \mathrm{hBN}$, and $\mathrm{MoS}_{2}$ homointerfaces. After complete retraction of the tip, our scanning electron microscopy (SEM) and AFM inspection of the lower section of the nanomesas on the $2 \mathrm{D}$ crystal substrate reveals an atomically flat and defect-free surface at the separation plane (top panel of Fig. 1b, c). From retraction force measurements (Fig. 1b), we observe a relatively gradual reduction of the adhesion force (rather than a snap-back to zero force), in particular, at the $\mathrm{G}$ and $\mathrm{hBN}$ homointerfaces, which looks at the first glance, fairly similar to the AFM rupture force curves of capillary nanobridges. However, the hydrophobic nature of $2 \mathrm{D}$ crystal nanomesas along with our $F-d$ approach curves, which display a small jump-to-contact force of $8-12 \mathrm{nN}$ at a small relative tip-sample distance of 5-6 nm (see, for instance, hBN/hBN approach curve in Fig. 1b), suggest dry contact at the interface with negligible effect of tip-sample capillary forces on the retraction curves. For comparison purposes, we recorded the rupture force curve of a water nanomeniscus formed between the in situ flattened $\mathrm{Si}$ tip and the $\mathrm{SiO}_{x} / \mathrm{Si}$ substrate at the relative humidity $(\mathrm{RH})$ of $70 \%$ (red curve in Fig. 1d). By closer inspection of the $F-d$ curves, we notice three fundamental differences in the separation mechanism between 2DLMs (e.g., blue curve in Fig. 1d) and capillary nanobridges (e.g., red curve in Fig. 1d). First, the separation range in the 2DLMs (typically $5-10 \mathrm{~nm}$ ) is almost an order of magnitude shorter than that in the capillary nanobridges (typically $50-80 \mathrm{~nm}$ ), further supporting the claim that the short-range vdW interaction (rather than the long-range nanobridge deformation) is the major separation mechanism in 2DLMs. Second, contrary to the case of the capillary nanobridges where the adhesion strongly depends on the retraction speed of the piezo ${ }^{22}$, our $F-d$ analysis under various tip retraction rates in the range of $1-1000 \mathrm{~nm} / \mathrm{s}$ reveals no appreciable effect on the IAE of 2DLMs, indicative of the absence of any dynamic (viscous) forces in the separation mechanism of 2DLMs. Third, an abrupt drop in the retraction force curves of nanobridges just prior to the complete separation could reflect the pinch-off process of the unstable meniscus neck, whereas a relatively fast transition from surface contact to line contact during the separation process in 2DLMs can eventually lead to the sudden break of the line contact and thereby an abrupt force drop at the very end of the separation process.

We also note that our shear force measurements (Fig. 1c) exhibit fluctuations in plateau regions for all 2D crystals, which can be attributed to the stick-slip friction of the tip-attached top mesa on the mobile bottom mesa, indicating that the present axial shear force microscopic technique can provide the shear force resolution up to the subnano level compared to the conventional lateral shear force microscopic technique. This is due to the fact that the spring constant of the probe in the axial shear force microscopy $\left(8.60 \mathrm{~N} \mathrm{~m}^{-1}\right)$ is an order of magnitude smaller than that in the conventional lateral shear force microscopy (83.8 $\mathrm{N} \mathrm{m}^{-1}$; Supplementary Fig. 9).

Figure le shows the intrinsic IAE (i.e., cohesion energy) of G, $\mathrm{hBN}$, and $\mathrm{MoS}_{2}$ crystals at room temperature with an average value of, respectively, $0.328 \pm 0.028,0.326 \pm 0.026$, and $0.482 \pm$ $0.032 \mathrm{~J} \mathrm{~m}^{-2}$ using normal force microscopy, matching well with the corresponding average value of $0.361 \pm 0.014,0.372 \pm 0.015$, and $0.501 \pm 0.017 \mathrm{~J} \mathrm{~m}^{-2}$ using the shear force microscopy. Slightly larger IAE values obtained by the shear measurement technique might be attributed to the small contribution of friction forces to the overall interfacial shear strength of $2 \mathrm{D}$ crystals. Nonetheless, both IAE measurement techniques indicate that the strongest interaction occurs between adjacent $\mathrm{MoS}_{2}$ layers due to dipolar, partially ionic Mo-S bonds, whereas nonpolar $\mathrm{C}-\mathrm{C}$ bonds and highly polar B-N bonds offer a roughly similar level of interaction at the $\mathrm{G}$ and hBN homointerfaces, respectively. In fact, stronger interactions with a faster detachment at the homointerface of $\mathrm{MoS}_{2}$ than $\mathrm{G}$ and $\mathrm{hBN}$ suggest more electron sharing and thus stronger interlayer bonding in $\mathrm{MoS}_{2}$ beyond a simple vdW-only interaction. Similar to the adhesion behavior at the $\mathrm{MoS}_{2}$ homointerface, a sudden detachment of $2 \mathrm{D}$ crystal tips from metal substrates (e.g., $\mathrm{Ni}, \mathrm{Cu}, \mathrm{Pt}$, and $\mathrm{Au}$ ) is observed with strong interfacial adhesion (see, for instance, the $F-d$ curve of $\mathrm{G}$ tip on the $\mathrm{Cu}$ substrate in Fig. 1d), suggesting that metal atoms share electrons with carbon atoms.

It is also worth making a comparison between our IAE results and those in the literature. There are several experimental methods to measure the IAE of $\mathrm{G}$ crystal with the reported values ranging from 0.15 to $0.72 \mathrm{~J} \mathrm{~m}^{-2}$ (see Supplementary Table 1). Among few direct measurements of intrinsic IAE values, we found that our measurements for cohesion energy of $G$ crystal are in excellent agreement with micro-force sensing probe measurements on $4-\mu \mathrm{m}$-wide square mesas $\left(0.37 \pm 0.01 \mathrm{~J} \mathrm{~m}^{-2}\right.$, ref. $\left.{ }^{15}\right)$ and AFM-assisted shearing measurements on 3- $\mu \mathrm{m}$-wide square mesas $\left(0.35 \mathrm{~J} \mathrm{~m}^{-2}\right.$, ref. $\left.{ }^{16}\right)$ but inconsistent with the AFMassisted shearing measurements on circular mesas of 100-600 nm in diameter $\left(0.227 \pm 0.005 \mathrm{~J} \mathrm{~m}^{-2}\right.$, ref. $\left.{ }^{17}\right)$. We revisited the lateral stiffness calibration of all probes used in ref. ${ }^{17}$ by means of a three-dimensional (3D) finite element simulation, predicting consistently stiffer ( $\sim 1.5$ times) probes than those described in the 
a

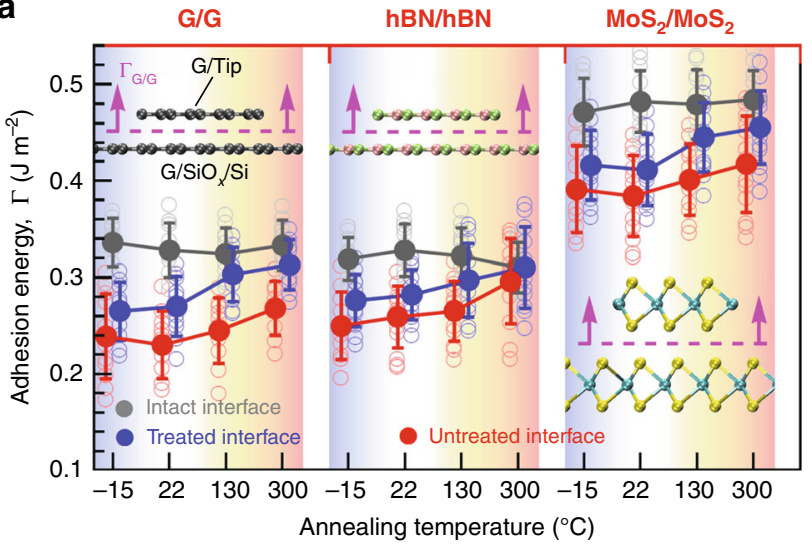

b

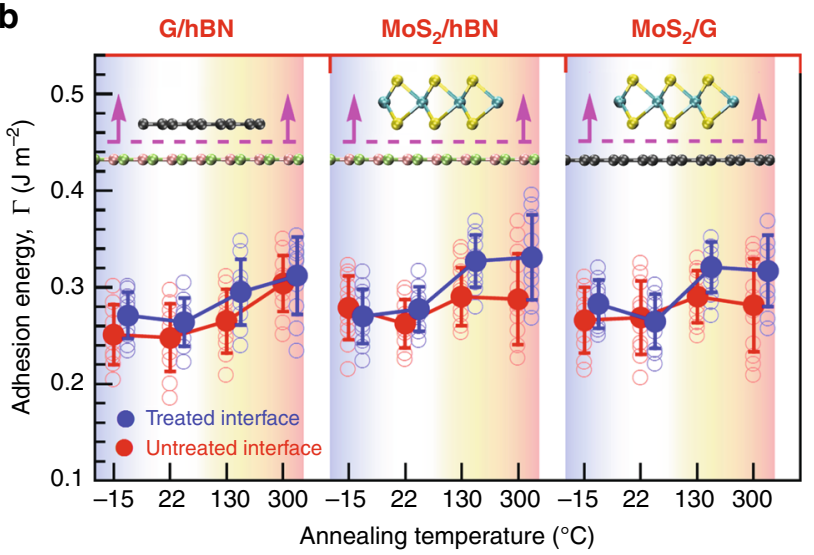

C

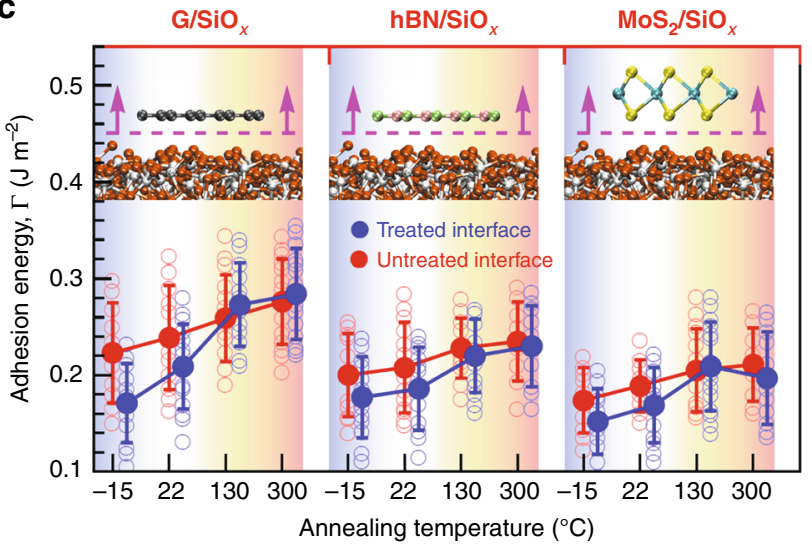

original work (Supplementary Fig. 11). Using this modified lateral spring constant yields an IAE value of $0.348 \pm 0.008 \mathrm{~J} \mathrm{~m}^{-2}$, more consistent with our measurements. We also note that, to the best of our knowledge, no IAE measurement on the hBN homointerface yet exists, while there is only one measured IAE value of $0.22 \mathrm{~J} \mathrm{~m}^{-2}$ at the $\mathrm{MoS}_{2}$ homointerface using a nanomechanical cleavage technique ${ }^{18}$, which is much lower than our values. Given that the bending stiffness of TMDs is reported in the range of $10-16 \mathrm{eV}^{23}$, we believe that a very low bending stiffness value of $0.92 \mathrm{eV}$ used in their calculations for the monolayer $\mathrm{MoS}_{2}$ has resulted in such a low IAE value.

Adhesion between similar vdW heterostructures. Figure 2 presents IAE values of both fresh and aged vdW heterostructures at the intact, precooling-treated and untreated heterointerfaces as a function of annealing temperatures. We begin our discussion
Fig. 2 Interfacial adhesion energy measurements. IAE values as a function of annealing temperatures at the $\mathbf{a} \mathrm{G}, \mathrm{hBN}$, and $\mathrm{MoS}_{2}$ homointerfaces; $\mathbf{b} \mathrm{G}$ / $\mathrm{hBN}, \mathrm{MoS}_{2} / \mathrm{hBN}$, and $\mathrm{MoS}_{2} / \mathrm{G}$ heterointerfaces; and c G/SiO${ }_{x}, \mathrm{hBN} / \mathrm{SiO}_{x}$ and $\mathrm{MoS}_{2} / \mathrm{SiO}_{x}$ heterointerfaces using normal force microscopy technique. Filled gray circles in a denote the intrinsic IAE values at the intact $G, h B N$, and $\mathrm{MoS}_{2}$ homointerfaces, whereas filled blue (red) circles in a-c denote the IAE values between 2D crystal tips and precooling-treated (untreated) substrates. Each open transparent gray circle in a represents a single IAE measurement at the intact homointerfaces, whereas each data point shown in open transparent blue and red circles in $\mathbf{a}, \mathbf{b}$ represents the IAE of the tips on an individual 2D crystal flake averaged over ten measurements from different locations of the flake surface. Similarly, each data point shown in open transparent blue and red circles in $\mathbf{c}$ represents the average IAE value obtained from 10 measurements within an individual small region $(1 \mu \mathrm{m} \times 1$ $\mu \mathrm{m})$ of $\mathrm{SiO}_{x}$ substrate. Each filled circle in $\mathbf{a}-\mathbf{c}$ is presented as average of all corresponding open circles \pm standard deviation. Insets in a-c illustrate balland-stick representation of various tip/substrate interfaces where carbon, boron, nitrogen, molybdenum, sulfur, silicon, and oxygen atoms are shown in gray, green, pink, cyan, yellow, white, and orange, respectively.

with IAE measurements at the intact and aged $2 \mathrm{D}$ crystal homointerfaces under different annealing temperatures (Fig. 2a). It is evident from the gray circles in Fig. 2a (and also Supplementary Table 5) that, upon the attachment of nanomesas to the AFM tip after the thermal annealing, the measured cohesion energy at the intact homointerfaces is, within our experimental accuracy, independent of the annealing temperatures. However, after exposing the freshly exfoliated 2D crystal flakes to the ambient air, the IAE between similar vdW heterostructures (red circles in Fig. 2a) is consistently lower than their corresponding intrinsic value, mainly due to the possible adsorption of airborne contaminants (e.g., water and hydrocarbon molecules) onto the fresh surface of crystals, thereby reducing their overall free surface (Gibbs) energy. A 30 and 19\% drop in the IAE of G/G and hBN/ $\mathrm{hBN}$, respectively, at room temperature suggests that $\mathrm{G}$ is more influenced by the airborne contaminants than $\mathrm{hBN}$ of similar lattice structure with only slightly larger $(\sim 1.8 \%)$ lattice constant. Although a mild annealing temperature $\left(130^{\circ} \mathrm{C}\right)$ coupled with relatively strong vdW interactions at the interface can provide a sufficient driving force to push the trapped water molecules away from the contact interface and thus to slightly improve the IAE of the crystals (up to $\sim 5 \%$ ), a higher annealing temperature is required to build up larger pressure at the interface to drive out the majority of hydrocarbons as the main source of such IAE drop, leading to nearly full recovery of the intrinsic IAE only at the $\mathrm{hBN}$ homointerface upon annealing at $300^{\circ} \mathrm{C}$. Interestingly, despite stronger vdW interaction of $\mathrm{MoS}_{2} / \mathrm{MoS}_{2}$ and similar level of interaction of $\mathrm{G} / \mathrm{G}$ compared to $\mathrm{hBN} / \mathrm{hBN}$, the full aggregation of such contaminants into nanobubbles at the $\mathrm{G}$ and $\mathrm{MoS}_{2}$ homointerfaces can only be triggered at a much elevated temperature, implying that hydrocarbons have a stronger interaction with $\mathrm{G}$ and $\mathrm{MoS}_{2}$ than hBN.

We next perform a series of measurements to study the effect of precooling treatment of the substrate on the IAE of the homointerfaces (blue circles in Fig. 2a). Surprisingly, such a precooling treatment can significantly improve the IAE of the $\mathrm{hBN}, \mathrm{G}$, and $\mathrm{MoS}_{2}$ crystals regardless of the subsequent annealing temperature. While such an IAE improvement upon $130{ }^{\circ} \mathrm{C}$ and $300^{\circ} \mathrm{C}$ thermal annealing could be intuitively understood by hypothesizing that the formation of ice-like monolayer/bilayer on the freshly cleaved 2D crystals can be effectively leveraged as a self-release underlying film for the facile removal of the subsequent hydrocarbon adsorptions, this hypothesis might not be supported by our findings at room temperature and $-15^{\circ} \mathrm{C}$ 
where the ice-like layer is still stable and tightly bonded to the underlying crystal surface. Notably, however, our observations can be fully supported for all range of temperatures by a recent study, showing that water adsorption on graphitic surfaces can significantly slow down the hydrocarbon adsorption rate ${ }^{24}$, thus making the nanometer-thick ice-like water an excellent protective layer against the airborne contamination for several hours. We also note that an increase of the annealing temperature from 130 to $300^{\circ} \mathrm{C}$ exhibits further improvement of the IAE, implying that the ice-like layer that is completely removed at $T \leq 130^{\circ} \mathrm{C}$ cannot fully cover the crystal surface, still leaving unprotected areas with adsorbed high boiling point hydrocarbons.

Adhesion between dissimilar vdW heterostructures. Our IAE measurements on dissimilar vdW heterostructures exposed to air at room temperature (red circles in Fig. 2b) reveal that the adhesion level at the untreated $\mathrm{G} / \mathrm{hBN}$ interface remains roughly the same as that at the untreated $\mathrm{G}$ and hBN homointerfaces over the temperature range of $-15-300^{\circ} \mathrm{C}$, whereas the IAE value of $\mathrm{MoS}_{2}$ on the untreated $\mathrm{G}$ and hBN substrates is considerably smaller than that on the untreated $\mathrm{MoS}_{2}$ substrate. During the approach-retract course, we observe a relatively stronger adhesive response of the $G$ nanomesa to $G$ than $h B N$ substrate within our experimental accuracy, suggesting that the $\mathrm{IAE}$ of $\mathrm{G} / \mathrm{hBN}$ is governed by a lower level of dispersion energy at the interface with a negligible contribution from the electrostatic interactions of $\mathrm{hBN}$, which are absent at the G/G interface. However, this is not the case at the contact interface between $\mathrm{MoS}_{2}$ and $\mathrm{hBN}(\mathrm{G})$ where different crystal structures and different static polarizabilities of the constituent atoms dictate very different levels of short-range dispersive (vdW) and long-range electrostatic (Coulombic) interactions at the $\mathrm{MoS}_{2} / \mathrm{MoS}_{2}$ and $\mathrm{MoS}_{2} / \mathrm{hBN}(\mathrm{G})$ interfaces. Such a different interaction energy level is further confirmed by recent cross-sectional scanning transmission electron microscopy imaging of $\mathrm{vdW}$ heterostructure interfaces, showing different interlayer separations between $\mathrm{MoS}_{2} / \mathrm{hBN}$ and $\mathrm{MoS}_{2} / \mathrm{MoS}_{2}{ }^{25}$.

Notably, we observe that, unlike very high-quality interface of untreated $\mathrm{MoS}_{2} / \mathrm{MoS}_{2}$ upon annealing at $300^{\circ} \mathrm{C}$, the untreated $\mathrm{MoS}_{2} / \mathrm{hBN}(\mathrm{G})$ interface does not show any further improvement, implying an absent or even negative impact of such a high annealing temperature on the IAE of $\mathrm{MoS}_{2} / \mathrm{hBN}(\mathrm{G})$. This counterintuitive observation can be explained by a trade-off between interface self-cleansing mechanisms driven by the vdW forces and $\mathrm{MoS}_{2}$ oxidation process triggered by relatively high temperatures $\left(>130^{\circ} \mathrm{C}\right)$ in the ambient air. On one hand, considerably weaker vdW interaction in $\mathrm{MoS}_{2} / \mathrm{hBN}(\mathrm{G})$ than in $\mathrm{MoS}_{2} / \mathrm{MoS}_{2}$ can still provide sufficient driving forces of similar magnitude to those of $\mathrm{G} / \mathrm{G}, \mathrm{hBN} / \mathrm{hBN}$, and $\mathrm{G} / \mathrm{hBN}$ for the segregation of the contaminants to the localized nanobubbles, leading to the enhanced IAE of $\mathrm{MoS}_{2} / \mathrm{hBN}(\mathrm{G})$ at $300^{\circ} \mathrm{C}$. On the other hand, the weaker interaction of $\mathrm{MoS}_{2} / \mathrm{hBN}(\mathrm{G})$ can facilitate the oxygen interfacial diffusion and thereby the oxidation process, which is initiated from the edges, grain boundaries, and intrinsic atomic defects of $\mathrm{MoS}_{2}$ and gradually penetrates into the $\mathrm{MoS}_{2}$ grains at the interface. This is consistent with the lowtemperature surface oxidation of $\mathrm{MoS}_{2}$, which is initiated at $\sim 100{ }^{\circ} \mathrm{C}$ and significantly increases at $300{ }^{\circ} \mathrm{C}^{26}$, resulting in the negative impact of oxygen adsorption on the mobility and homogeneity of $\mathrm{MoS}_{2} / \mathrm{G}$ heterostructure devices after annealing above $150^{\circ} \mathrm{C}^{27}$. We hypothesize two possible interfacial oxidation mechanisms responsible for the weaker interaction of $\mathrm{MoS}_{2} / \mathrm{hBN}$ (G) at $300^{\circ} \mathrm{C}$ : (1) replacement of sulfur atoms with oxygen atoms results in a lower surface energy in the oxidized $\mathrm{MoS}_{2}\left(\mathrm{MoO}_{3}\right)$ than unreacted $\mathrm{MoS}_{2} ;(2)$ partial protrusions $\left(0.36 \pm 0.25 \mathrm{~nm}^{28}\right)$ at the interface due to formation of interfacial $\mathrm{MoO}_{3}$ patches along with the presence of gaseous reaction products (e.g., $\mathrm{SO}_{2}$, which cannot diffuse out of interface owing to very high vdW pressure on the trapped molecular layers ${ }^{29}$ ) can give rise to local interlayer decoupling of unreacted $\mathrm{MoS}_{2}$ crystal from underlying hBN and $\mathrm{G}$ substrates.

Similar to the precooling-treated $\mathrm{G}, \mathrm{hBN}$, and $\mathrm{MoS}_{2}$ homointerfaces, it is evident from the blue circles in Fig. $2 \mathrm{~b}$ that precooling treatments can effectively protect the crystal substrates against the airborne contaminants and thus boost the adhesion level at the interface of dissimilar vdW heterostructures at much lower annealing temperature of $130{ }^{\circ} \mathrm{C}$. However, such a protective layer offers no appreciable improvement in the IAE of $\mathrm{MoS}_{2} / \mathrm{hBN}(\mathrm{G})$ at $300^{\circ} \mathrm{C}$, further confirming the possible destructive effect of interfacial contaminations/oxygen diffusion on the $\mathrm{MoS}_{2}$ oxidation at higher temperatures (see Supplementary Fig. 10 for our X-ray photoelectron spectroscopy (XPS) on both freshly exfoliated and pre-annealed $\mathrm{MoS}_{2}$ samples).

During the revision of this paper, $\mathrm{Li}$ et al. reported the vdW interactions of $\mathrm{G} / \mathrm{hBN}$ and $\mathrm{G} / \mathrm{MoS}_{2}$ heterostructures, where a $\mathrm{Si}$ AFM tip wrapped with a thin $\mathrm{G}$ flake is brought into contact with pre-annealed $\mathrm{G}, \mathrm{hBN}$, and $\mathrm{MoS}_{2}$ substrates in high vacuum at room temperature ${ }^{19}$. Using a G-wrapped sharp tip with an unknown contact area only allows the measurements of the critical adhesion forces (i.e., pull-off forces, $P$ ) between $\mathrm{G} / \mathrm{G}, \mathrm{G} /$ $\mathrm{hBN}$, and $\mathrm{G} / \mathrm{MoS}_{2}$. Qualitatively speaking, their measurements showed that $\mathrm{G}$ experiences a weaker vdW interaction with $\mathrm{hBN}$ and $G$ than $\mathrm{MoS}_{2}$, yielding a critical adhesion force ratio of $P_{\mathrm{G} / \mathrm{MoS}_{2}} / P_{\mathrm{G} / \mathrm{hBN}}=1.079$ and $P_{\mathrm{G} / \mathrm{MoS}_{2}} / P_{\mathrm{G} / \mathrm{G}}=1.028$. Similarly, our IAE ratios of $\Gamma_{\mathrm{G} / \mathrm{MoS}_{2}} / \Gamma_{\mathrm{G} / \mathrm{hBN}}$ and $\Gamma_{\mathrm{G} / \mathrm{MoS}_{2}} / \Gamma_{\mathrm{G} / \mathrm{G}}$ for the roughly similar experimental conditions (i.e., precooling-treated heterointerfaces annealed at $130^{\circ} \mathrm{C}$ ) are 1.088 and 1.059 , respectively, which are qualitatively in good agreement with their findings.

Adhesion between 2D crystals and $\mathrm{SiO}_{x}$. Despite many experimental and theoretical studies devoted to the IAE determination of $2 \mathrm{D}$ crystals $/ \mathrm{SiO}_{x}$ heterostructures, no experimental data, to our knowledge, are available on the interaction of $\mathrm{hBN} / \mathrm{SiO}_{x}$, whereas the reported IAE data on the interaction of $\mathrm{G}$ and $\mathrm{MoS}_{2}$ with $\mathrm{SiO}_{x}$ are very diverse, ranging from 0.14 to $0.90 \mathrm{~J} \mathrm{~m}^{-2}$ at the $\mathrm{G} / \mathrm{SiO}_{x}$ interface (Supplementary Table 3 ) and $0.17-0.48 \mathrm{~J} \mathrm{~m}^{-2}$ at the $\mathrm{MoS}_{2} / \mathrm{SiO}_{x}$ interface (Supplementary Table 4). To gain an atomiclevel understanding of interaction mechanisms at the 2D crystals/ $\mathrm{SiO}_{\mathrm{x}}$ heterointerfaces, we first report in Fig. 2c an AFM quantitative characterization of the interlayer interactions of 2D crystals on both untreated and precooling-treated $\mathrm{SiO}_{x}$ substrates. Similar to untreated $2 \mathrm{D}$ crystals, the thermal annealing can effectively remove the water and hydrocarbons from the untreated $\mathrm{SiO}_{x}$ surface, leading to the higher IAE at the $2 \mathrm{D}$ crystal $/ \mathrm{SiO}_{x}$ interface. Surprisingly, however, unlike the case of $2 \mathrm{D}$ crystal substrates, the precooling treatment results in the weaker interaction of the $2 \mathrm{D}$ crystals with the $\mathrm{SiO}_{x}$ substrate at both $-15^{\circ} \mathrm{C}$ and room temperature. This weaker interaction may be explained by the hydrophilic nature of $\mathrm{SiO}_{x}$ that can adsorb a homogeneous and flat water film of thickness $2-3 \mathrm{~nm}$ ( 6-10 monolayers of water) on its silanol ( $\mathrm{Si}-\mathrm{OH})$-rich surface when storing at $-15^{\circ} \mathrm{C}$ (corresponding to $\left.100 \% \mathrm{RH}\right)^{30}$. As such, our IAE measurements at $-15^{\circ} \mathrm{C}$ (i.e., $0.171 \pm 0.041,0.177 \pm 0.042$, and 0.152 $\pm 0.034 \mathrm{~J} \mathrm{~m}^{-2}$ for the $\mathrm{G}, \mathrm{hBN}$, and $\mathrm{MoS}_{2}$ crystals on $\mathrm{SiO}_{x}$, respectively) essentially take place at the $2 \mathrm{D}$ crystal/water interface rather than at the $2 \mathrm{D}$ crystal $/ \mathrm{SiO}_{x}$ interface. In addition, a larger IAE of $2 \mathrm{D}$ crystal/treated $\mathrm{SiO}_{x}$ at room temperature compared to that at $-15^{\circ} \mathrm{C}$ can be attributed to the presence of a mixture of ice-like monolayer/bilayer structure (fully H-bonded 

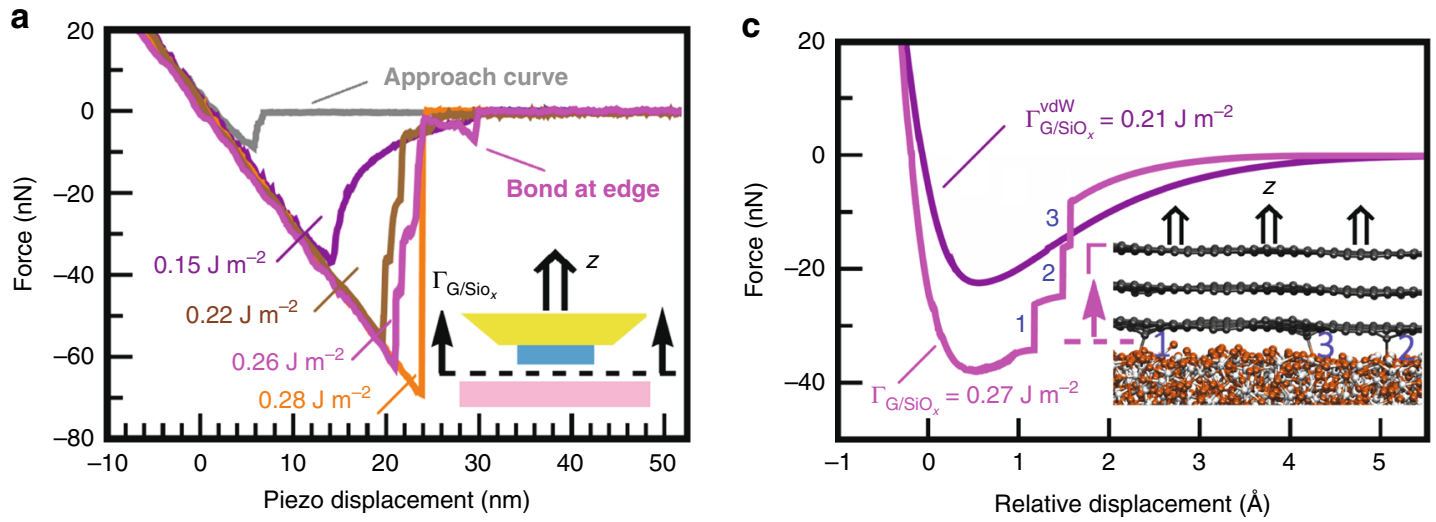

b

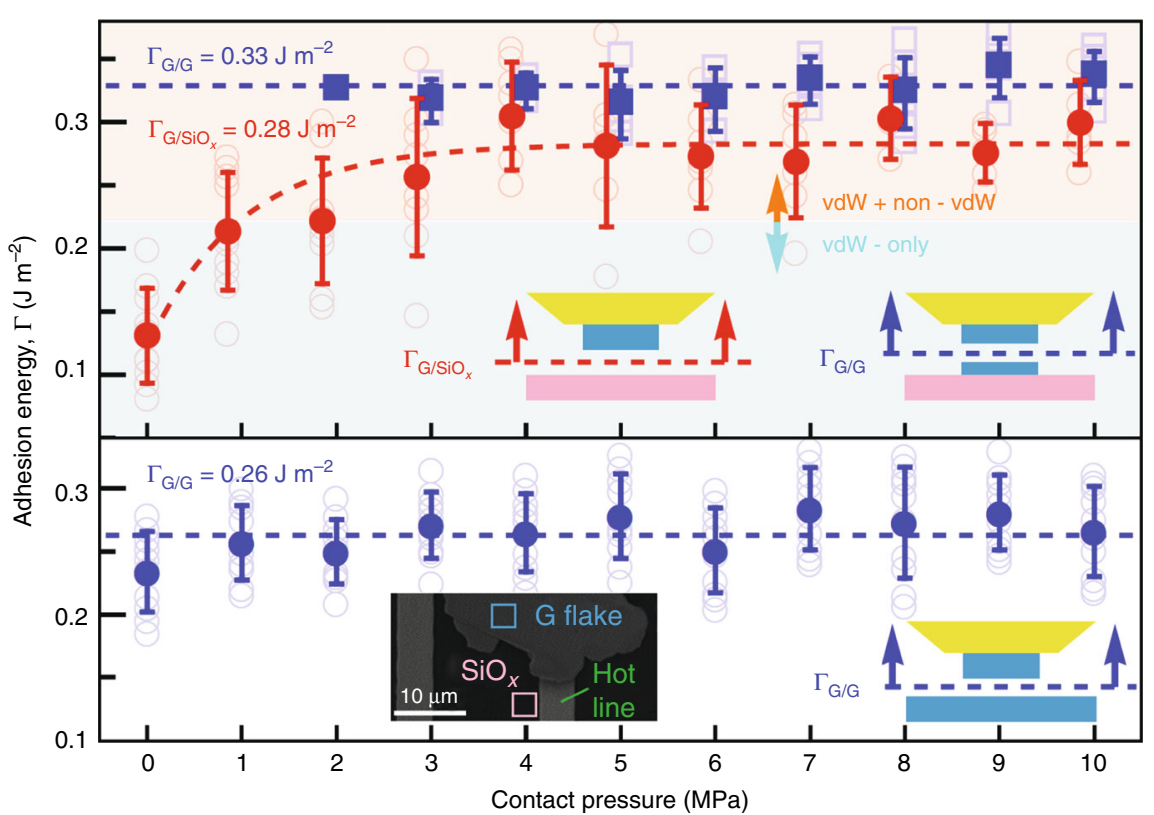

Fig. 3 Interaction mechanism at $\mathbf{G} / \mathbf{S i O}_{\mathbf{x}}$ heterointerface. a Typical retraction $\mathrm{F}$ - $d$ curves of the $\mathrm{G} / \mathrm{SiO}_{x}$ interface recorded at no contact pressure (in purple) and $5 \mathrm{MPa}$ (in brown, magenta and orange) and also a typical approach $F$-d curve (in gray). $\mathbf{b} \mid \mathrm{AE}$ values as a function of contact pressure at the intact $\mathrm{G}$ homointerfaces (filled blue squares in top panel), $\mathrm{G} / \mathrm{SiO}_{x}$ interfaces (filled red circles in top panel), and aged $\mathrm{G}$ homointerfaces (filled blue circles in bottom panel). Each open transparent symbol represents a single IAE measurement at the given contact pressure. Each filled symbol is presented as average of all corresponding open symbols \pm standard deviation. From 110 IAE measurements shown in the top panel, 33 and 77 contacts result in the separation of $\mathrm{G}$ crystal tip across the thickness of the nanomesa (open blue squares) and from the $\mathrm{SiO}_{x}$ surface (open red circles), respectively. In the top panel, cyan and orange shaded regions indicate the vdW-only and vdW+non-vdW interactions of $\mathrm{G} / \mathrm{SiO}_{x}$, respectively, where the lift-off in the vdW-only region is relatively gradual in comparison to the vdW+non-vdW region. Dashed blue lines denote an overall average IAE value of $0.328 \pm 0.022$ and 0.263 $\pm 0.032 \mathrm{~J} \mathrm{~m}^{-2}$ at the intact $\mathrm{G}$ homointerface (top panel) and aged $\mathrm{G}$ homointerface (bottom panel), respectively. Dashed red line in the top panel represents the best fit to the data, indicating the pressure dependence of IAE at the interface with an average value of $0.284 \pm 0.046 \mathrm{~J} \mathrm{~m} \mathrm{~m}^{-2}$ taken within the pressure-independent region (i.e., $\geq 3 \mathrm{MPa}$ ). Inset of bottom panel: SEM image showing $2 \mu \mathrm{m} \times 2 \mu \mathrm{m}$ square regions of $\mathrm{G}$ (in turquoise) and $\mathrm{SiO} \mathrm{O}_{x}$ (in rose) substrates on which all measurements are performed in close proximity to the microheater. $\mathbf{c} M D$-calculated force versus relative displacement curves for the vdW-only interaction of $\mathrm{G} / \mathrm{SiO}_{x}$ (in purple) and vdW+non-vdW interaction of $\mathrm{G} / \mathrm{SiO}_{x}$ (in magenta) with three covalent $\mathrm{C}$-O-Si bonds at the interface. Each force jump labeled by 1, 2, and 3 represents the break of the corresponding covalent bond, as illustrated by the MD pull-off simulation in the inset.

to the silanol groups) and liquid-like few-layer structure on top of the ice-like layer at room temperature. Such trapped liquid-like film can segregate into isolated nano-sized bubbles ${ }^{31}$ by means of the contact pressure and the interlayer vdW forces, bringing the $2 \mathrm{D}$ crystals into closer proximity with the $\mathrm{SiO}_{x}$ surface and thus enhancing the IAE at the $2 \mathrm{D}$ crystal $/ \mathrm{SiO}_{x}$ interface. We also note that the thermal annealing at 130 and $300{ }^{\circ} \mathrm{C}$ makes no appreciable difference in the interfacial adhesion level between $2 \mathrm{D}$ crystal/precooling-treated $\mathrm{SiO}_{x}$ and $2 \mathrm{D}$ crystal/untreated $\mathrm{SiO}_{x}$, confirming the formation of the protective ice-like layer on the untreated $\mathrm{SiO}_{x}$ substrate due to $15 \% \mathrm{RH}$ in the ambient air.
Similar to the interaction of $\mathrm{MoS}_{2}$ crystal with the other 2D crystals, the high annealing temperature of $300{ }^{\circ} \mathrm{C}$ can reduce the adhesion level at the $\mathrm{MoS}_{2} /$ treated $-\mathrm{SiO}_{x}$ interface, which lends additional support to the hypothesis of $\mathrm{MoS}_{2}$ oxidation at higher temperatures due to the chemical reaction of $\mathrm{MoS}_{2}$ with the trapped water and the diffused oxygen. Such significantly reduced IAE of $\mathrm{MoS}_{2} /$ treated $\mathrm{SiO}_{x}$ relative to $\mathrm{MoS}_{2} / \mathrm{hBN}(\mathrm{G})$ at $300^{\circ} \mathrm{C}$ can be understood as a direct consequence of the strong hydrophilic property of $\mathrm{SiO}_{x}$, where the $\mathrm{MoS}_{2}$ crystal undergoes an additional chemical reaction with the interfacial water layer, resulting in the partial etching of the $\mathrm{MoS}_{2}$ interface layer and needle-like 
protrusions due to the formation of $\mathrm{MoO}_{3} \cdot \mathrm{H}_{2} \mathrm{O}$ on the $\mathrm{MoS}_{2}$ surface $^{32}$. It is worth pointing out that although oxygen plasma treatment can completely remove any water and hydrocarbon molecules, leaving a contamination-free $\mathrm{SiO}_{x}$ surface terminated with more silanol groups, we observed that stronger interaction between $2 \mathrm{D}$ crystals and plasma-cleaned $\mathrm{SiO}_{x}$ surface leads to the exfoliation of $2 \mathrm{D}$ crystals across the thickness of nanomesa, making a direct IAE measurement at $2 \mathrm{D}$ crystals/plasma-treated $\mathrm{SiO}_{x}$ interfaces inaccessible.

Adhesion at G/SiOx interface: beyond vdW interaction. During interfacial adhesion measurements of $\mathrm{G} / \mathrm{SiO}_{x}$ at the annealing temperatures of 130 and $300{ }^{\circ} \mathrm{C}$, we unexpectedly observed abrupt detachment of $\mathrm{G}$ nanomesa with single/multiple force jumps in the $F-d$ retraction curves, resulting in much stronger interfacial interactions in $\mathrm{G} / \mathrm{SiO}_{x}$ than in $\mathrm{hBN}\left(\mathrm{MoS}_{2}\right) / \mathrm{SiO}_{x}$. In particular, the separation process of $2 \mathrm{D}$ crystal tips in our set-up dictates a relatively gradual reduction of the interfacial adhesion force between two adjacent $2 \mathrm{D}$ crystal flakes and between $\mathrm{hBN}\left(\mathrm{MoS}_{2}\right)$ nanomesas and $\mathrm{SiO}_{x}$ substrate. As a result, such sudden detachment with single/multiple plateau force jumps in the $\mathrm{G} / \mathrm{SiO}_{x}$ adhesion curves cannot be interpreted as a consequence of experimental noises, thermal fluctuations, and mechanical instabilities of the probe, as they are roughly equally present in all our $F-d$ measurements.

From over hundred interfacial adhesion measurements for the $\mathrm{G} / \mathrm{SiO}_{x}$ interaction, we identified three distinct $F-d$ curves, each describing gradually broken contacts (i.e., weak interaction without any force jump), suddenly broken contacts (i.e., strong interaction with a single force jump), and a transition from gradually to suddenly broken contacts (i.e., mild interaction with multiple force jumps), as shown in Fig. 3a. To provide a rational explanation of the origin of such distinctive interfacial behavior in the $\mathrm{G} / \mathrm{SiO}_{x}$ heterostructure, we begin by addressing quantitatively to what degree the interfacial adhesion of $\mathrm{G} / \mathrm{SiO}_{x}$ interfaces (and also intact and aged $\mathrm{G}$ homointerfaces for comparative purposes) is controlled by the conformity of the tip-attached $\mathrm{G}$ nanomesa to the underlying substrate morphology. To this end, a series of interfacial adhesion measurements over a pressure range of $0-10$ $\mathrm{MPa}$ was conducted at the interface of $\mathrm{G}$ crystal tip/pre-annealed $\mathrm{SiO}_{x}$ substrate (top panel of Fig. $3 \mathrm{~b}$ ) and $\mathrm{G}$ crystal tip/preannealed $\mathrm{G}$ substrate (bottom panel of Fig. $3 \mathrm{~b}$ ). This set-up only allowed us to study the interaction of $\mathrm{G} / \mathrm{SiO}_{x}$ weaker than that between G/G (red circles in Fig. 3b), otherwise the separation takes place across the thickness of $G$ nanomesa (blue squares in Fig. 3b).

It is observed from Fig. 3b (top panel) that the G crystal tip requires a contact pressure of $\geq 3 \mathrm{MPa}$ to conform closely to the $\mathrm{SiO}_{x}$ surface, thereby enhancing the IAE of the $\mathrm{G} / \mathrm{SiO}$ interface from $0.131 \pm 0.038 \mathrm{~J} \mathrm{~m}^{-2}$ at zero pressure to $0.289 \pm 0.034 \mathrm{~J} \mathrm{~m}^{-2}$ at $10 \mathrm{MPa}$. In contrast, both the intact $\mathrm{G}$ homointerface (blue squares in the top panel of Fig. 3b) and the aged G homointerface (bottom panel in Fig. 3b) suggest a constant IAE value of $0.328 \pm$ 0.022 and $0.263 \pm 0.032 \mathrm{~J} \mathrm{~m}^{-2}$, respectively, almost entirely independent of the pressure, indicative of flat and dangling bond-free G/G interfaces. It is also evident from the top panel in Fig. $3 b$ that graphene flakes are not exfoliated from the tipattached $\mathrm{G}$ nanomesa onto the $\mathrm{SiO}_{x}$ at very low pressure $(<2$ $\mathrm{MPa}$ ) and only 10 and $20 \%$ of contacts at 2 and $3 \mathrm{MPa}$, respectively, result in the exfoliation of graphene flakes, indicating the significant contribution of the conformal adhesion to the overall interfacial adhesion strength of the $\mathrm{G} / \mathrm{SiO}_{x}$ interface. More importantly, we observe that abrupt detachment events with single/multiple force jumps in the retraction curves of $\mathrm{G} / \mathrm{SiO}_{x}$ take place more frequently at higher pressure in such a way that nearly all contacts are suddenly broken at the pressure load of $\geq 4$ $\mathrm{MPa}$ with IAE values roughly $>0.221 \mathrm{~J} \mathrm{~m}^{-2}$. Surprisingly, this value is very close to theoretical calculations of the intrinsic vdW interaction energy $\left(0.230 \mathrm{~J} \mathrm{~m}^{-2}\right)$ at the $\mathrm{G} / \mathrm{SiO}_{x}$ interface obtained for the multilayer graphene blister tests on the $\mathrm{SiO}_{x}$ substrate under pressure loading ${ }^{20}$. Hence, while a continuous decrease in the retraction curves can be attributed to the long-range vdW interaction of $\mathrm{G} / \mathrm{SiO}_{x}$ with IAE values typically $<0.221 \mathrm{~J} \mathrm{~m}^{-2}$, direct observation of single/multiple force jumps at stronger G/ $\mathrm{SiO}_{x}$ interfaces can be hypothesized to be the result of formation of short-range chemical bonds at the interface. Both experimental and theoretical results confirm that $\mathrm{G}$ flakes supported on $\mathrm{SiO}_{x}$ exhibits much higher chemical reactivity than suspended $G$ flakes, mainly due to the combined action of inhomogeneously distributed charge puddles (induced by polar adsorbates, such as water molecules on the silanol surface and by ionized impurities, such as $\mathrm{Na}^{+}$ions trapped on $\mathrm{SiO}_{x}$ ) and larger topographic corrugations (induced by thermal fluctuation and $\mathrm{vdW}$ interaction at the $\mathrm{G} / \mathrm{SiO}_{x}$ interface $)^{33-35}$. As such, hydrogen and oxygen molecules preferentially bind to apexes of corrugated $G$ due to the combined contribution from the enhanced elastic and electronic energies of convex regions on the $G$ surface ${ }^{36}$.

In addition, the specific chemical reactivity of the carbon atoms with accessible and highly active electrons at the edge of the G flakes can also contribute to the formation of chemical bonds, as observed in a number of our retraction curves (e.g., see the magenta curve in Fig. 3a and also see Supplementary Note 2.6). This, coupled with our molecular dynamics (MD) observations that force jumps in the retraction curves can only be achieved by breaking short-range chemical bonds at the $\mathrm{G} / \mathrm{SiO}_{x}$ interface (Fig. 3c), provides further support for the hypothesis that more likely hydrogen bonds and/or less likely covalent bonds are formed between $\mathrm{G}$ and $\mathrm{SiO}_{x}$. Notably, from Fig. 3b, only $\sim 22 \%$ chemical bond-induced improvement in the $\mathrm{IAE}$ of $\mathrm{G} / \mathrm{SiO}{ }_{x}$ (i.e., from $0.221 \pm 0.030$ to $0.284 \pm 0.046 \mathrm{~J} \mathrm{~m}^{-2}$ ) under relatively low pressure (of the order of few MPa) leads us to believe that (1) vdW interactions are still the dominant mechanism of adhesion at the $\mathrm{G} / \mathrm{SiO}_{x}$ interface; and (2) the formation of hydrogen bonds (e.g., C-H...O-Si, C-O...H-O-Si, and C-O-H...O-Si in the absence of contaminants and $\mathrm{C}-\mathrm{H} . . . \mathrm{O}_{\backslash \mathrm{H}}-\mathrm{H}$... O-Si and $\mathrm{C}-\mathrm{O} \ldots$ $\mathrm{H}-\mathrm{O}-\mathrm{H} . . \mathrm{O}-\mathrm{Si}$ in the presence of water molecules) rather than covalent bonds could result in such force jumps in the retraction curves; nonetheless, the formation of any covalent bonds between $\mathrm{G}$ and $\mathrm{SiO}_{x}$ (e.g., $\mathrm{C}-\mathrm{O}-\mathrm{Si}$ and $\mathrm{C}-\mathrm{Si}^{37,38}$ ) cannot be completely ruled out because the effect of localized tensile strain and charge transfer on the chemical activity level of the corrugated $G$ is poorly understood.

It is also to be noted that, in contrast to MD-calculated retraction curves in Fig. 3c, the number of force jumps in Fig. 3a does not necessarily correspond to the number of chemical bonds at the $\mathrm{G} / \mathrm{SiO}_{x}$ interface. One may argue from an interfacial fracture standpoint that, once the restoring force of the probe cantilever exceeds the strength of the $\mathrm{G} / \mathrm{SiO}_{x}$ interaction (i.e., the pull-off force), interfacial nano-sized cracks start to form due to the localized nano delamination across the separation plane and propagate until complete interfacial fracture occurs ${ }^{2}$. As such, the interfacial fracture of $\mathrm{G} / \mathrm{SiO}_{x}$ heterostructure is a combined action of the external pull-off force and the internal adhesion force (i.e., $\mathrm{vdW}$ and/or non-vdW forces). For the case of the vdW-only interaction of $\mathrm{G} / \mathrm{SiO}_{x}$, both a smaller pull-off force and the smooth and slow propagation of nanocracks contribute to the relatively gradual reduction of the interfacial adhesion force (purple curve in Fig. 3a). In contrast, faster crack propagation in the stronger vdW+non-vdW interaction of $\mathrm{G} / \mathrm{SiO}_{x}$, which is triggered by a larger pull-off force, results in the abrupt force drop 
a
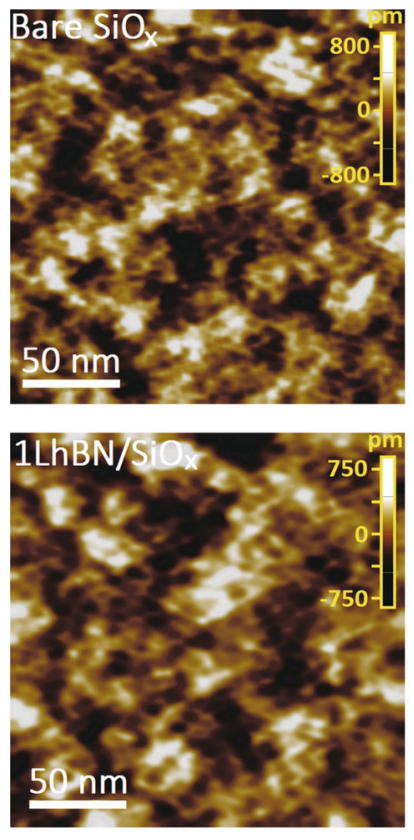
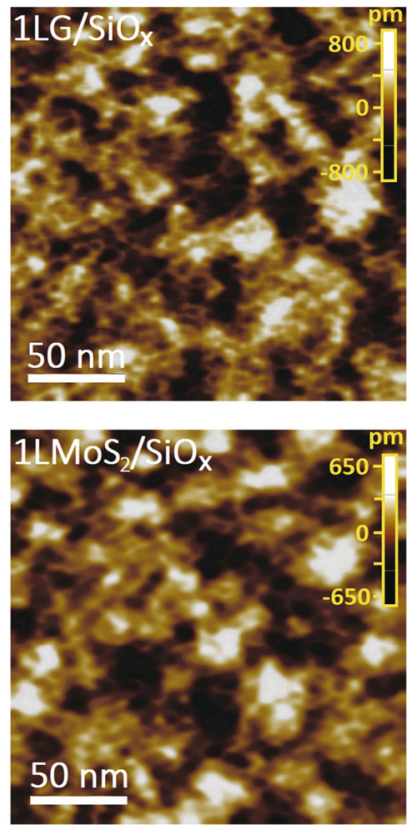

b
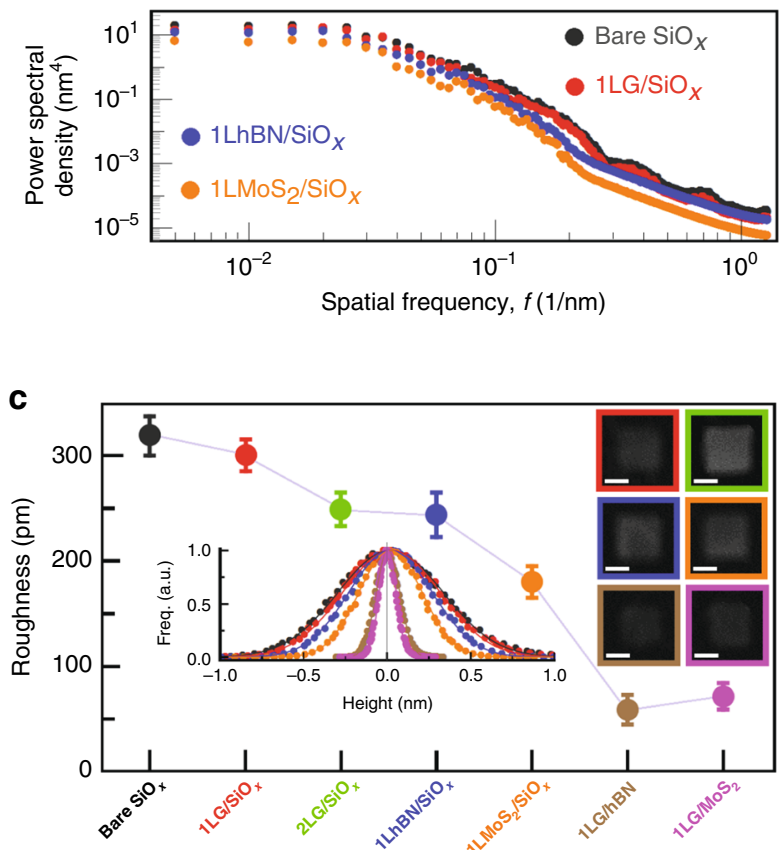

Fig. 4 Surface roughness measurements of different heterostructures. a High-resolution AFM images of the surface roughness of bare $\mathrm{SiO}_{x}$, monolayer graphene (1LG), monolayer hBN (1LhBN), and monolayer $\mathrm{MoS}_{2}\left(1 \mathrm{LMoS}_{2}\right)$ on $\mathrm{SiO}_{x}$ substrate. b PSD profiles corresponding to the images in a. c Surface roughness measurements of different heterostructures. Error bars show the spread of data over several independent measurements of different flakes. Left inset: histogram of the height distribution (surface roughness) for bare $\mathrm{SiO}_{x}, 1 \mathrm{LG} / \mathrm{SiO}_{x}, 1 \mathrm{LhBN} / \mathrm{SiO}_{x}, 1 \mathrm{LMoS} 2 / \mathrm{SiO}$, $1 \mathrm{LG} / \mathrm{hBN}$, and $1 \mathrm{LG} / \mathrm{MoS} \mathrm{Substrates}$ Solid lines are Gaussian fits to the distribution. Right inset: representative SEM images of 2D crystal square mesas exfoliated onto the substrate. Scale bar in each is $5 \mu \mathrm{m}$. b, c and the insets of $\mathbf{c}$ share the same color legend.

in the retraction curves immediately upon the initiation of the separation process. As the nanocracks are continuously propagating and the pull-off force becomes progressively smaller and smaller, the chemical bonds (i.e., the anchoring spots) gain the ability to pin the nanocrack tips and thus momentarily retard the crack propagation. Such unique crack arresting behavior at the G/ $\mathrm{SiO}_{x}$ interface gives rise to very short signals in the retraction curves through a significant decrease in the force drop rate, making the detection of the chemical bonds possible in our set-up (brown and magenta curves in Fig. 3a). However, in the case of suddenly broken contacts with a single force jump (e.g., orange curve in Fig. 3a), as the number of the interfacial chemical bonds increases, larger and larger pull-off forces are required to initiate the interfacial fracture of the $\mathrm{G} / \mathrm{SiO}_{x}$, thereby much faster nanocrack propagation at the beginning of the separation process causes all interfacial chemical bonds to suddenly break and thus no longer allows our set-up to capture the crack-arresting behavior during propagation. Furthermore, while both the pulloff and interfacial adhesion forces are primarily responsible for developing the interfacial nanocrack growth and separation (see step-like events in the retraction curves), when the pull-off force approaches zero, further pull-off force needs to build up to overcome possible chemical bonds at the edge of $G$ flakes (magenta curve in Fig. 3a).

Origin of distinctive interfacial adhesion behavior in $\mathrm{G} / \mathrm{SiO}$. To gain a sub-nanoscale insight into the origin of the distinctive interfacial behavior in the $\mathrm{G} / \mathrm{SiO}_{x}$ heterostructure specifically and into the underlying interaction mechanism of $2 \mathrm{D}$ crystals and $\mathrm{SiO}_{x}$ in general, we perform 3D surface topographic measurements of single-layer $2 \mathrm{D}$ crystals (transfer-printed on the $\mathrm{SiO}_{x}$ surface under a controlled contact pressure of $5 \mathrm{MPa}$ ) with the power spectral density (PSD) analysis of the surface roughness data (Supplementary Note 3). Typical high-resolution $200 \times 200$ $\mathrm{nm}^{2}$ AFM topographic images of bare $\mathrm{SiO}_{x}$ and monolayer $\mathrm{G}$, $\mathrm{hBN}$, and $\mathrm{MoS}_{2}$ flakes supported on $\mathrm{SiO}_{x}$ and the PSD profiles corresponding to the images are shown in Fig. 4a, b, respectively. It is evident that highly random corrugations with sub-nanometer vertical dimension but few-nanometer lateral dimension in monolayer $2 \mathrm{D}$ crystals are imposed by the underlying $\mathrm{SiO}_{x}$ substrate. In Fig. 4c, the average surface roughness of monolayer 2D crystal/ $/ \mathrm{SiO}_{x}$ heterostructures and histograms of the corresponding height distribution are presented, where the measurements from the bilayer of $\mathrm{G}$ on $\mathrm{SiO}_{x}$ and monolayer of $\mathrm{G}$ on the hBN and $\mathrm{MoS}_{2}$ substrates are also shown for comparative purposes. Our roughness measurements suggest that monolayer $\mathrm{G}$ exhibits the highest degree of conformation to the $\mathrm{SiO}_{x}$ (roughness ratio: $0.94)$, followed by bilayer $\mathrm{G}(0.78)$, monolayer hBN (0.76), and monolayer $\mathrm{MoS}_{2}$ (0.57). As expected, the topography of monolayer $\mathrm{G}$ on $\mathrm{hBN}$ and $\mathrm{MoS}_{2}$ substrates is much smoother than that of monolayer $\mathrm{G}$ on $\mathrm{SiO}_{x}$, suggesting an atomically flat contact at $\mathrm{G} / \mathrm{hBN}$ and $\mathrm{G} / \mathrm{MoS}_{2}$ interfaces. Assuming that the conformation of $2 \mathrm{D}$ crystal flakes to the underlying $\mathrm{SiO}_{x}$ substrate of similar corrugation pattern is proportional to their IAE but inversely proportional to the bending stiffness of the flakes ${ }^{39}$ with a value of $D_{1 \mathrm{LG}}=1.49 \mathrm{eV}, D_{2 \mathrm{LG}}=35.5 \mathrm{eV}, D_{1 \mathrm{LhBN}}=1.34 \mathrm{eV}$, and $D_{1 \mathrm{LMoS}_{2}}=11.7 \mathrm{eV}$, (Supplementary Note 7 ), the smaller bending stiffness of monolayer hBN compared to monolayer and bilayer $\mathrm{G}$, however, results in a smoother surface morphology, further confirming the stronger IAE at the $\mathrm{G} / \mathrm{SiO}_{\mathrm{x}}$ interface. Moreover, our comparative study of the corrugation of bilayer $G$ and monolayer $\mathrm{MoS}_{2}$ with almost the same thickness (i.e., $0.670 \mathrm{~nm}$ in 2LG versus $0.645 \mathrm{~nm}$ in $1 \mathrm{LMoS}_{2}$ ) also demonstrates that the adhesion of bilayer $\mathrm{G}$ to $\mathrm{SiO}_{x}$ is much stronger than that of 
Table 1 Cohesion energy $(\Gamma)$, intrinsic cleavage strength $\left(\sigma_{\mathrm{s}}\right)$ and intrinsic interlayer shear strength $\left(\tau_{\mathrm{s}}\right)$ of 2D crystals.

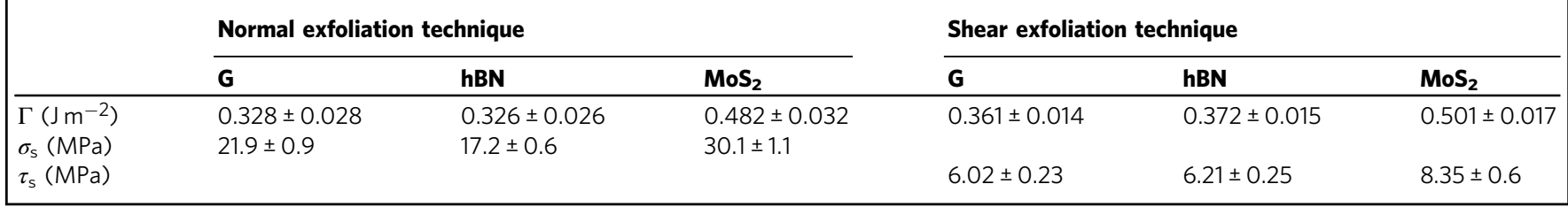

monolayer $\mathrm{MoS}_{2}$. Such intimate and strong interaction of $\mathrm{G} / \mathrm{SiO}{ }_{x}$ suggests that the electron-scattering sites across the interface as well as the convex sites of corrugated $G$ result in the formation of short-range chemical bonds, which act as anchoring spots to locally pin $\mathrm{G}$ to the $\mathrm{SiO}_{x}$ surface at the location of such chemically active sites ${ }^{33-36}$. Since monolayer $\mathrm{G}$ with high flexibility possesses more chemically active sites than multilayer $\mathrm{G}$ at the $\mathrm{G} / \mathrm{SiO}_{x}$ interface, stronger adhesion energy of monolayer to $\mathrm{SiO}_{x}$ is expected, as previously confirmed by a pressurized blister test to be $0.45 \pm 0.02 \mathrm{~J} \mathrm{~m}^{-2}$ for monolayer $\mathrm{G}$ but $0.31 \pm 0.03 \mathrm{~J} \mathrm{~m}^{-2}$ for multilayer $\mathrm{G}^{21}$.

\section{Discussion}

While the IAE is obtained from the information of all points on the $F-d$ curve, which provides valuable insight into the whole separation process, cleavage strength and interlayer shear strength of $2 \mathrm{D}$ crystals (Table 1) can be obtained from the information of one single point on the $F-d$ curve (i.e., the pull-off force in Fig. $1 \mathrm{~b}$ and the maximum shear force in Fig. 1c). Thanks to the known contact area in our set-up, the intrinsic cleavage strength of $\mathrm{G}, \mathrm{hBN}$, and $\mathrm{MoS}_{2}$ crystals at room temperature is measured to be $21.9 \pm 0.9,17.2 \pm 0.6$, and $30.1 \pm 1.1 \mathrm{MPa}$, respectively. Our average value for the cleavage strength of $\mathrm{G}$ is consistent with that (10.3-20.7 MPa) reported using static tests for polycrystalline graphite ${ }^{40}$. Also, the interlayer shear strength of $\mathrm{G}, \mathrm{hBN}$, and $\mathrm{MoS}_{2}$ crystals for a nanomesa of width/diameter $\sim 60 \mathrm{~nm}$ is calculated to be $6.02 \pm 0.23,6.21 \pm 0.25$, and $8.35 \pm 0.6$ $\mathrm{MPa}$, respectively. Given that the interlayer shear strength is inversely proportional to the diameter (width) of the circular (square) mesas (see Supplementary Note 2.1), our values are in good agreement with those obtained by the AFM-assisted shearing technique for a $50-\mathrm{nm}$-radius $\mathrm{G}$ nanomesa $(3.1 \mathrm{MPa})^{17}$ and microforce shearing technique for a 12.6-nm-width $\mathrm{MoS}_{2}$ nanomesa $(25.3 \pm 0.6 \mathrm{MPa})^{41}$.

We already explained in Fig. 3a the origin of different trends in our $F-d$ curves for the $\mathrm{G} / \mathrm{SiO}_{x}$ heterostructure by means of interfacial fracture mechanics. Similarly, we believe that, for the case of the relatively weak vdW-only interaction (e.g., hBN/hBN in Fig. 1b), both a smaller pull-off force and the smooth and slow propagation of nanocracks contribute to the relatively gradual reduction of the interfacial adhesion force. In contrast, faster crack propagation in the relatively stronger vdW-only interaction (e.g., G/G in Fig. 1b), which is triggered by a larger pull-off force, results in the abrupt force drop in the retraction curves immediately upon the initiation of the separation process. However, in the case of suddenly broken contacts (e.g., $\mathrm{MoS}_{2} / \mathrm{MoS}_{2}$ in Fig. $1 \mathrm{~b}$ and $\mathrm{G} / \mathrm{Cu}$ in Fig. 1d), the more electron sharing at the interface, the larger pulloff force is required to initiate the interfacial fracture, thereby much faster nanocrack propagation at the beginning of the separation process causes a sudden break of the contact.

Although we showed in Fig. 2b, c that $\mathrm{MoS}_{2}$ has a higher chemical activity than $G$, in particular, at higher temperatures, short-range chemical bonds are not formed at the $\mathrm{MoS}_{2} / \mathrm{SiO}_{\mathrm{x}}$ interface. A possible reason is that, unlike the $\mathrm{G}$ crystal, the basal plane of $\mathrm{MoS}_{2}$ is rather inert unless $S$ vacancies are introduced into its basal plane ${ }^{42}$. Short-range chemical reactions between
$\mathrm{MoS}_{2}$ and $\mathrm{SiO}_{x}$ require (1) vacancy defects in the $\mathrm{MoS}_{2}$ basal plane to directly bind $\mathrm{H}, \mathrm{O}$, and $\mathrm{Si}$ atoms to exposed Mo atoms and (2) a close conformation of $\mathrm{MoS}_{2}$ to the underlying $\mathrm{SiO}_{x}$ substrate. For the former one, our surface topographic measurements in the absence of the thermal annealing do not exhibit vacancy defects and grain boundaries in the basal plane of $\mathrm{MoS}_{2}$ nanomesas. For the latter one, we showed that the degree of conformation of $\mathrm{MoS}_{2}$ to the $\mathrm{SiO}_{x}$ is much lower than that of $\mathrm{G}$ (Fig. 4). These two reasons make the formation of the chemical bonds at the $\mathrm{MoS}_{2} / \mathrm{SiO}_{x}$ interface almost impossible at least when the contact forms at room temperature, as is the case in Fig. 3 for the $\mathrm{G} / \mathrm{SiO}_{x}$ heterostructures.

In order to provide a valuable guideline for the fabrication of vdW heterostructures based on the vdW pick-up transfer techniques, we present a summary of the cohesion energy at the intact $\mathrm{G}, \mathrm{hBN}$, and $\mathrm{MoS}_{2}$ homointerfaces (Supplementary Table 5) and the IAE of untreated and precooling-treated homostructures/ heterostructures (Supplementary Table 6), corresponding to the experimental data points in Figs. 1e and 2, respectively. These tables reveal that hBN may not successfully pick up $\mathrm{G}$ flakes from $\mathrm{G}$ and $\mathrm{MoS}_{2}$ substrates, whereas the strong adhesion at the $\mathrm{MoS}_{2} /$ $\mathrm{hBN}(\mathrm{G})$ interface makes $\mathrm{MoS}_{2}$ a better candidate for the selective $\mathrm{G}$ and hBN pick-up from the $\mathrm{G}$ and hBN substrates. These tables also suggest that both $\mathrm{G}$ and $\mathrm{MoS}_{2}$ can be used to pick up all three $2 \mathrm{D}$ crystals from the $\mathrm{SiO}_{x}$ substrate at room temperature. However, the stronger adhesion of $\mathrm{G}$ to the $\mathrm{SiO}_{x}$ substrate requires careful selection of the $2 \mathrm{D}$ crystals for the high-yield $\mathrm{G}$ pick-up, making hBN a relatively improper choice for such a purpose at room temperature. Moreover, the simple precooling treatment of the $\mathrm{SiO}_{x}$ substrate before the mechanical exfoliation of $2 \mathrm{D}$ crystals can highly facilitate the $2 \mathrm{D}$ crystal pick-up by reducing the IAE at the $2 \mathrm{D}$ crystal $/ \mathrm{SiO}_{x}$ interface.

Since the effect of airborne surface contaminations and thermal annealing on the IAE of $2 \mathrm{D}$ crystals can be well understood by contact angle measurements, we herein discuss the interaction of $2 \mathrm{D}$ crystals with airborne contaminants and quantify the effect of surface contaminations and thermal annealing on the IAE of $2 \mathrm{D}$ crystals by characterizing intrinsic water wettability of fresh and aged surfaces of $2 \mathrm{D}$ crystals with a focus on the G crystal. Many studies on the wettability of $G$ crystal along with very limited studies on the wettability of hBN and $\mathrm{MoS}_{2}$ crystals all suggest that freshly cleaved crystals spontaneously adsorb airborne contamination upon the air exposure, leading to an increase in the water contact angle (WCA) of $\mathrm{G}^{43}, \mathrm{hBN}^{44}$, and $\mathrm{MoS}_{2}{ }^{45}$ crystals, respectively, from $\left(64^{\circ}, 63^{\circ}, 69^{\circ}\right)$ measured within few seconds of air exposure of fresh surfaces to a saturated value of $\left(90^{\circ}, 86^{\circ}, 89^{\circ}\right)$ within few hours of air exposure. Our further analysis on the temporal evolution of the adhesion energy (see Supplementary Note 6 for our detailed analysis) and contamination thickness measured on the mechanically exfoliated $G$ surface during the first $60 \mathrm{~min}$ of air exposure reveals that its intrinsic IAE of 0.341 $\pm 0.025 \mathrm{~J} \mathrm{~m}^{-2}$ obtained under ultrahigh vacuum or ultrahighpurity argon atmosphere is well consistent with our experimental value of $0.328 \pm 0.028 \mathrm{~J} \mathrm{~m}^{-2}$ but drastically decreases within the first minute of air exposure and eventually approaches a saturated value of $0.15 \pm 0.02 \mathrm{~J} \mathrm{~m}^{-2}$ after $10 \mathrm{~min}$ (Fig. 5), which is smaller 


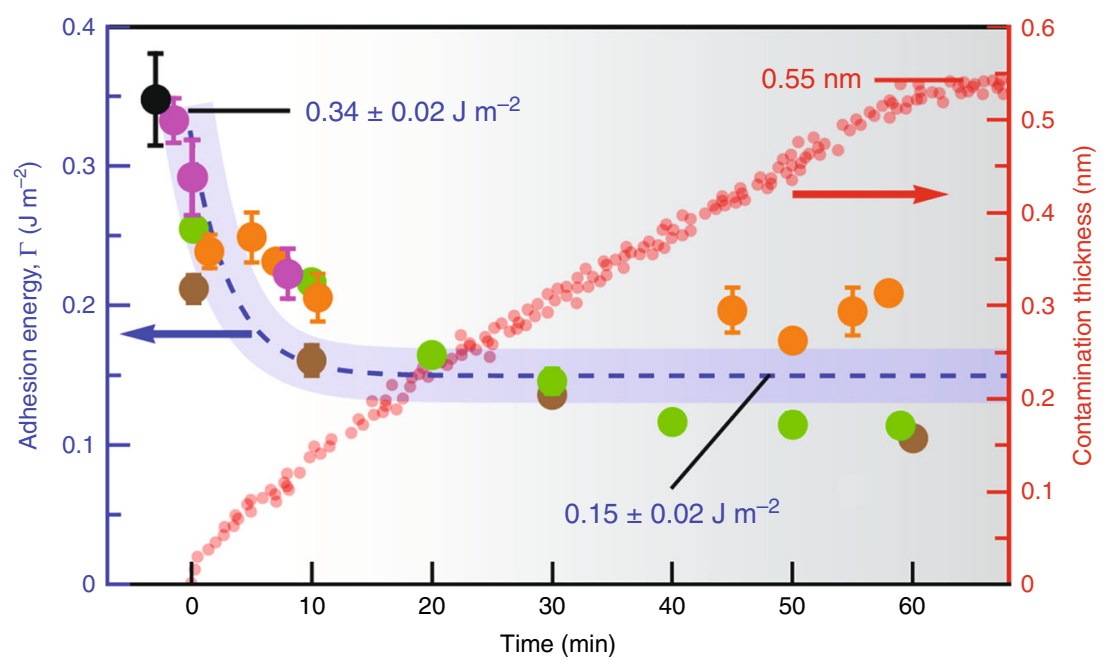

Fig. 5 Adhesion energy evolution in G crystal. Temporal evolution of the adhesion energy (left axis in blue) and contamination thickness (right axis in red) measured on the mechanically exfoliated HOPG surface during the first $60 \mathrm{~min}$ of air exposure. Adhesion energy is extracted from WCA measurements of ref. ${ }^{46}$, ref. ${ }^{47}$, ref. ${ }^{48}$, ref. ${ }^{49}$, and ref. ${ }^{24}$ denoted by black, brown, orange, magenta, and green circles, respectively. Adsorbed contamination layers linearly grow within the first $60 \mathrm{~min}$ of air exposure, reaching a thickness of $\sim 0.55 \mathrm{~nm}$, and then the growth rate considerably decreases and plateaus at $\sim 0.60 \mathrm{~nm}$ after several hours 24 .

than our IAE value of $0.233 \pm 0.035 \mathrm{~J} \mathrm{~m}^{-2}$ upon roomtemperature storage for $1 \mathrm{~h}$. This could be attributed to the presence of the contact pressure and the vdW interaction between the layers in our experiments, which may still play a role to squeeze away the contaminants even at room temperature, leaving cleaner interfaces with stronger interactions. We also note that a substantial decrease in the surface hydrocarbon level under vacuum, high-temperature $\left(500-1000^{\circ} \mathrm{C}\right)$ treatment during the WCA measurements results in the IAE recovery of the G crystal $\left(0.282 \pm 0.024 \mathrm{~J} \mathrm{~m}^{-2}\right)$, which is in good agreement with our IAE value of $0.268 \pm 0.028 \mathrm{~J} \mathrm{~m}^{-2}$ at much lower temperature $\left(300^{\circ} \mathrm{C}\right)$, further confirming the dominant contribution of the $\mathrm{vdW}$ force to the IAE improvement.

In conclusion, we have used an AFM-assisted nanomanipulation technique to directly and precisely measure the weak interlayer vdW bonding at the fresh and aged vdW homointerfaces/ heterointerfaces. Highly stronger interactions at the homointerface of $\mathrm{MoS}_{2}$ than G and hBN suggested possible sharing electrons in the interlayer region of TMDs beyond a simple vdWonly interaction. After quantifying the effect of airborne contaminants and humidity on the interfacial adhesion level, we revealed to what degree contaminated heterointerfaces can recover their IAE upon thermal annealing through precise temperature control of nanocontact interfaces. We showed that the precooling treatments can significantly improve the interfacial adhesion of the $\mathrm{hBN}, \mathrm{G}$, and $\mathrm{MoS}_{2}$ crystals regardless of the subsequent annealing temperature. Our combined experimental and atomistic analysis also suggested that the formation of shortrange chemical bonds only in $\mathrm{G} / \mathrm{SiO}_{x}$ heterostructures can elucidate the mechanistic origin of the distinctive strong adhesion behavior between $\mathrm{G}$ and $\mathrm{SiO}_{x}$ beyond the widely accepted vdW interaction. Our precise nanoscale quantification of weak interlayer vdW bonding in 2D materials and vdW heterostructures not only provides a reliable basis for theoretical calculations but also can be of fundamental and technological importance for the mass production and continued development of such promising materials in modern electronic devices.

\footnotetext{
Methods

AFM-assisted experimental set-up. All AFM measurements were performed under controlled ambient conditions $\left(T=22^{\circ} \mathrm{C}\right.$ and $\left.15 \% \mathrm{RH}\right)$ by a Park XE-70
}

microscope, which was isolated from mechanical floor vibration by a microscope vibration isolator and also from acoustic vibration, ambient light disturbance, and air flow by a closed box. We determined the noise floor of our AFM set-up to be consistently $<0.3 \AA$ throughout the measurements. Three small pieces of $\mathrm{SiO}_{\mathrm{x}} / \mathrm{Si}$ substrate were simultaneously loaded onto the AFM stage, including $2 \mathrm{D}$ crystal flakes mechanically exfoliated with adhesive tape on microheater arrays that are prefabricated on the $\mathrm{SiO}_{x} / \mathrm{Si}$ substrate (piece\#1), 25-nm-thick polymer glue (poly (3,4-ethylenedioxythiophene):poly(styrene sulfonate) with D-sorbitol (PEDOT:PSS (D-sorbitol))) coated on the $\mathrm{SiO}_{x} / \mathrm{Si}$ substrate (piece\#2), and pre-patterned bulk 2D crystal stamps with 50-100-nm-thick square and circular nanomesas of 55-65 nm in width and diameter, respectively (piece\#3). To minimize the effect of the relative tilt angle, all three pieces were attached to a larger piece of $\mathrm{SiO}_{x} / \mathrm{Si}$ substrate precoated by the ultrathin glue film, followed by placing the larger piece onto a multistage Peltier cooling element equipped with a tilt control mechanism (angle resolution: $\pm 0.5^{\circ}$ ) beneath the cooling stage (Supplementary Note 1).

Surface preparation of 2D crystal flakes. Instead of immediately removing all $2 \mathrm{D}$ crystal-loaded adhesive tapes from piece\# 1 to complete the mechanical exfoliation onto the microheaters, we only peeled off the tape containing the $2 \mathrm{D}$ crystal flakes of interest $\left(\mathrm{G}, \mathrm{hBN}\right.$, or $\left.\mathrm{MoS}_{2}\right)$ for the interfacial adhesion measurements, thereby enabling much better control over the possible adsorption of airborne contaminants onto the fresh surface of $2 \mathrm{D}$ crystals. We prepared aged substrates by two different aging conditions: (1) the freshly exfoliated 2D crystal substrate and the bare $\mathrm{SiO}_{x} / \mathrm{Si}$ substrate were directly exposed to the ambient air for $1 \mathrm{~h}$ at room temperature; (2) 2D crystal flakes were freshly exfoliated on a precooled $\left(-15^{\circ} \mathrm{C}\right)$ $\mathrm{SiO}_{x} / \mathrm{Si}$ substrate and the sample was kept at this temperature for $15 \mathrm{~min}$, followed by the air exposure of the exfoliated $2 \mathrm{D}$ crystal substrate and the underlying $\mathrm{SiO}_{x} / \mathrm{Si}$ substrate for $1 \mathrm{~h}$ at room temperature. A similar method was used to aged tipattached nanomesas for the subsequent contact with their corresponding aged substrates where fresh $2 \mathrm{D}$ crystal tips were simply obtained by our previously developed AFM-assisted shear exfoliation technique ${ }^{2}$ (Supplementary Note 1).

Fabrication of nano-sized 2D crystal mesas. An $\sim 100$-nm-thick bilayer of polymethyl methacrylate (PMMA) $495 \mathrm{~K}(60 \mathrm{~nm}) / 950 \mathrm{~K}(40 \mathrm{~nm})$ is spin coated onto the freshly cleaved surface of 1-mm-thick highly oriented pyrolytic graphite (HOPG) (SPI, Grade 1, with a mosaic spread value of $0.4^{\circ}$ ), hBN (grade A, with single-crystal domains over $100 \mu \mathrm{m})$, and $\mathrm{MoS}_{2}$ (429MS-AB, natural single crystals from Canada) substrates, baked each layer for $10 \mathrm{~min}$ at $120^{\circ} \mathrm{C}$ to evaporate the solvent, and then patterned by electron beam lithography. After developing the exposed PMMA area in 1:3 MIBK/NMP, a 10-nm-thick aluminum film is deposited by thermal evaporation, followed by lift-off process in acetone. The unprotected $\mathrm{HOPG}, \mathrm{hBN}$, and $\mathrm{MoS}_{2}$ areas are thinned down by using a reactive ion etching system with pure $\mathrm{O}_{2}$ (precursor flow rate: $10 \mathrm{sccm}$, RF power: $40 \mathrm{~W}$, pressure: $10 \mathrm{mTorr}), \mathrm{CHF}_{3} / \mathrm{Ar} / \mathrm{O}_{2}(10 / 5 / 2 \mathrm{sccm}, 30 \mathrm{~W}, 10 \mathrm{mTorr})$ and $\mathrm{SF}_{6}(20$ $\mathrm{sccm}, 100 \mathrm{~W}, 20 \mathrm{mTorr}$ ) reactive gases, respectively. Square (circular) mesas with a width (diameter) of 55-65 $\mathrm{nm}$ and etch depth of 50-100 $\mathrm{nm}$ emerge from 2D crystal substrates during the plasma etch. After plasma etching, the sample is soaked in $0.1 \mathrm{~mol} / 1 \mathrm{KOH}$ water solution for $\sim 3 \mathrm{~min}$ to remove the $\mathrm{Al}$ layer, followed by an annealing process at $600{ }^{\circ} \mathrm{C}$ under constant $\mathrm{Ar} / \mathrm{H}_{2}$ flow for $1 \mathrm{~h}$ to 
remove any resist/metallic residues from 2D crystal substrates (Supplementary Notes 1.1 and 1.2).

\section{Attachment of 2D crystal nanomesas to the AFM tip. For $F-d$ measurements, a} highly doped silicon AFM probe (NANOSENSORSTM, ATEC-FM, with a nominal spring constant of $2.8 \mathrm{~N} \mathrm{~m}^{-1}$ and a typical tip radius of curvature better than 10 $\mathrm{nm}$ ) was used where the tip is positioned at the very end of the cantilever and pointing outward, which provides a more accurate positioning of the tip apex. Since our experiments require a flat plateau at the apex parallel to the piece\# 1 surface on which all interfacial adhesion measurements were conducted, we scanned the tip in contact mode on its $\mathrm{SiO}_{x}$ surface to achieve an atomically flat surface with a root mean square (RMS) roughness of $<1 \mathrm{~nm}$. The in situ flattened tip was next moved from piece\#1 to piece\#2 and coated with a very thin layer of polymer glue by putting the tip apex in gentle contact with the PEDOT:PSS(Dsorbitol) film. For the precise attachment of $2 \mathrm{D}$ crystal nanomesa to the gluecoated flattened apex, the tip was moved from piece\# 2 to piece\#3, followed by locating the nanomesas by the non-contact AFM topographic measurements. The glue-coated tip apex was then moved to the center of the selected $2 \mathrm{D}$ crystal nanomesa and held in contact with the nanomesa for $10 \mathrm{~min}$. Afterwards, the tip was gently pulled away from the substrate surface in a direction perpendicular (parallel) to the single basal plane of $2 \mathrm{D}$ crystal, leading to pulling off (shearing) the upper section of the nanomesa (attached to the tip apex) from the lower section (fixed to the 2D crystal substrate) (Supplementary Note 1.3). Compared to 2D crystal micro-sized mesas, the tip-attached nano-sized mesas alone offered four striking features to our set-up: (1) the presence of a single-crystalline grain across the whole nanomesa is guaranteed, enabling an atomically defect-free contact interface; (2) substantially more reliable and robust IAE measurements can be achieved under any possible small relative tilting angle between the tip-attached mesa and the substrate, assuring perfect face-to-face contact during approach-retract tip manipulation (Supplementary Fig. 8); (3) high-resolution topographic images in non-contact mode can still be taken to locate $2 \mathrm{D}$ crystal flakes of interest for the subsequent IAE measurements (see the AFM image taken by the G crystal tip in the left inset of Fig. 1a); and (4) the nano-sized contact area with a significantly smaller interfacial adhesion force allows using the AFM probe with a lower spring constant and thus higher force resolution.

Temperature control of nanocontact interface. While an AFM Peltier-based cooling stage was used to probe the IAE of $2 \mathrm{D}$ crystal tips on substrate surfaces at subzero temperature $\left(-15^{\circ} \mathrm{C}\right)$, the IAE measurements at elevated temperatures (up to $300^{\circ} \mathrm{C}$ ) on the target substrates were conducted by means of localized Joule heating of ultralow power microheaters (Supplementary Notes 1.4 and 1.5). Using the microheater can not only significantly alleviate the adverse effect of high temperature on the AFM probe by locally heating the substrate (Supplementary Fig. 4) but also provide a uniform temperature distribution over the heated 2D flakes, which are in direct contact with the heating lines (Supplementary Figs. 5 and 6). For each temperature change, enough time was given to the $2 \mathrm{D}$ flakes to reach steady-state temperature. Then the $2 \mathrm{D}$ crystal tip was engaged with the sample surface at a pressure of $5 \mathrm{MPa}$ (unless otherwise noted) for $15 \mathrm{~min}$ to reach thermal equilibrium and then the substrate cooled back down to room temperature to perform the interfacial adhesion measurements. Similarly, we conducted a series of the interfacial adhesion measurements at subzero temperature by first cooling the substrate surface down to $-15^{\circ} \mathrm{C}$ using a multistage Peltier element and then removing the $2 \mathrm{D}$ crystal-loaded adhesive tape from piece\#1 (Supplementary Note 1.5).

Force-displacement measurements. All retraction $F-d$ curves between the 2D crystal tips and the untreated/precooling-treated substrates were obtained under controlled ambient conditions in the near-equilibrium regime, which was achieved by an ultralow noise floor of $<0.3 \AA$, an ultralow noise AFM controller with the $Z$ scanner's vertical resolution of better than $0.1 \AA$, and also using a very slow (quasistatic) pulling rate of $1 \mathrm{~nm} / \mathrm{s}$. Very careful adjustment of the $Z$ servo gain to suppress any possible oscillation of the $Z$ scanner could further make the retraction measurements in the near-equilibrium regime possible (Supplementary Note 2). In order to calculate the IAE per unit area $\left(\Gamma, \mathrm{J} \mathrm{m}^{-2}\right)$ from the recorded

retraction-displacement curves, we integrate the retraction force as a function of the piezo displacement (light blue-shaded area in Fig. 1b, d), followed by dividing the resulting adhesion energy by the known contact area at the interface. However, in order to extract the IAE from the shear $F-d$ curves, the interfacial adhesion force opposing new surface formation is first obtained as $F_{\mathrm{a}}(x)=\Gamma[\mathrm{d} A(x) / \mathrm{d} x]$, where $x$ represents the lateral displacement of the mobile section of the mesa with respect to the initial position, and $A(x)$ is the overlap area of the top and bottom sections of the mesa as a function of $x$. For a square mesa of width $w$ and a circular mesa of diameter $D$, the maximum shear force, $F_{\mathrm{s}}^{\mathrm{m}}$, in the shear $F-d$ curves that is required to initiate sliding (i.e., $F_{\mathrm{a}}$ at $x=0$ ) can be related to the IAE by $\Gamma w$ and $\Gamma D$, respectively (Supplementary Note 2.1 ). While the cleavage strength can be obtained by $\sigma_{33}=P / A$, where $P$ is the pull-off force and $A$ is the interface area, by definition of the interlayer shear strength at the sliding interface, $\tau_{\mathrm{s}}=F_{\mathrm{s}}^{\mathrm{m}} / A$ (Supplementary Note 2.1). The accuracy of $F-d$ measurements can be limited by the uncertainty in the determination of the interfacial contact area and spring constant of the AFM probe (Supplementary Note 2.2). In order to create a known contact area, we used $2 \mathrm{D}$ crystal tips with a very well-defined geometric shape parallel to the substrate, enabling an atomically clean and flat contact interface. Our interfacial adhesion measurements reveal that the tilting angle between the tip and the substrate is smaller than $1^{\circ}$, indicating perfect face-to-face contact during measurements (Supplementary Note 2.3). We reduced the second main source of uncertainty in our measurements by determining the stiffness of the AFM cantilever by means of three different methods and took their mean value as the static normal $(3.05 \pm 0.05$ $\left.\mathrm{N} \mathrm{m}^{-1}\right)$ and axial $\left(8.60 \pm 0.40 \mathrm{~N} \mathrm{~m}^{-1}\right)$ spring constants of the probe, suggesting a relative calibration error of 2 and 5\%, respectively (Supplementary Note 2.4). The same AFM probe was used throughout the experiments to ensure accurate cor relation between all interfacial adhesion measurements. The laser spot was also kept at the same position on the lever to avoid any changes in the force measurements. After performing all the measurements, the spring constant of the probe was again determined in ambient conditions to make sure that the cooling/ local annealing of the substrate has no appreciable effect on its stiffness, yielding the spring constant still within the uncertainty range of our measurements. We also note that the random crystalline orientation at the interface of $2 \mathrm{D}$ crystal tips and 2D crystal substrates has no appreciable effect on the IAE measurements (Supplementary Note 2.5). Our further analysis of $F-d$ curves also confirms the negligible effect of the possible edge functionalization of nanomesas (due to the etching process) on the IAE measurements (Supplementary Note 2.6).

Adhesion of $\mathbf{G} / \mathbf{S i O}_{\mathbf{x}}$ under different contact pressures. We conducted a series of interfacial adhesion measurements over a pressure range of $0-10 \mathrm{MPa}$ at the interface of $\mathrm{G}$ crystal tip/pre-annealed $\mathrm{SiO}_{x}$ substrate and $\mathrm{G}$ crystal tip/preannealed $G$ substrate. To further minimize experimental uncertainty, a $2 \mu \mathrm{m} \times 2$ $\mu \mathrm{m}$ smooth region of the $\mathrm{SiO}_{x}(\mathrm{G})$ substrate with an RMS surface roughness of $0.305 \mathrm{~nm}(0.077 \mathrm{~nm})$ was first located by non-contact AFM roughness measurements and then 10 contacts with 100 -nm interval spacing were formed at each pressure load under a very clean environment, allowing us to perform all measurements within a very small region in close proximity to the microheater (see the SEM image in the inset of bottom panel in Fig. 3b). Moreover, prior to each pressure increment, $\mathrm{SiO}_{x}$ and $\mathrm{G}$ substrates are annealed at $300{ }^{\circ} \mathrm{C}$ for $30 \mathrm{~min}$ to remove any possible adsorbed contaminations and then the $\mathrm{G}$ crystal tip/preannealed substrate interface is further annealed at $300^{\circ} \mathrm{C}$ for $15 \mathrm{~min}$, followed by the new round of adhesion measurements.

3D surface topography measurements. Monolayers of $\mathrm{G}, \mathrm{hBN}$, and $\mathrm{MoS}_{2}$ square flakes of $10 \mu \mathrm{m}$ in width were shear exfoliated from a flat polydimethylsiloxane stamp onto the pre-annealed $\mathrm{SiO}_{x}$ substrate at a contact pressure of $5 \mathrm{MPa}$. We used an ultrasharp tip with $2 \mathrm{~nm}$ nominal radius of curvature $(<5 \mathrm{~nm}$ guaranteed) and sparing constant of $39.1 \mathrm{~N} \mathrm{~m}^{-1}$ in the non-contact mode and in the attractive regime (with a frequency shift of $-10 \mathrm{~Hz}$ and free amplitude of $7.5 \mathrm{~nm}$ ) under ambient conditions and then determined the noise floor of the AFM system, being consistently $<0.3 \AA$. To provide a more accurate and comprehensive description of the surface roughness both in vertical and lateral directions, the AFM image data were analyzed by the 2D PSD function rather than the standard RMS roughness (Supplementary Note 3).

X-ray photoelectron spectroscopy. XPS measurements were performed on both freshly exfoliated and pre-annealed $\mathrm{MoS}_{2}$ samples at excitation energy of 1486.6 $\mathrm{eV}$. Residual electrostatic charging effects were taken into account by applying an offset to the spectra with a reference signal of C (1s) at a binding energy of 284.6 $\mathrm{eV}$. In order to eliminate any interference between the dominant Mo $(3 d)$ and $\mathrm{S}$ $(2 s)$ features, we used the less intense Mo $(3 p)$ photoelectron signal for the quantification purposes. Each set of peaks was fitted by a $70 \%$ Gaussian-30\% Lorentzian function. In addition, peaks of spin-orbit doublets Mo $\left(3 p_{3 / 2}\right.$ and $\left.3 p_{1 / 2}\right)$ were set to have an area ratio in accordance with quantum degeneracy values (i.e., $2: 1$ for $3 p_{3 / 2}$ and $3 p_{1 / 2}$ orbitals; Supplementary Note 4 ).

Classical MD simulations. To gain an in-depth understanding of underlying mechanisms associated with the interaction of $\mathrm{G}$ crystal and the $\mathrm{SiO}_{x}$ substrate, we performed classical MD simulations using the LAMMPS simulator at room temperature. Four $98.2 \AA \times 102.1 \AA \mathrm{G}$ layers with $\mathrm{AB}$ stacking were placed at a distance of $3.0 \AA$ above an amorphous $\mathrm{SiO}_{x}$ substrate $\left(143.3 \times 146.5 \times 21.3 \AA^{3}\right)$ while the flattened tip was modeled by a tapered silicon (001) layer. To hold the system in space, $2 \AA$ of the $\mathrm{SiO}_{x}$ substrate from the bottom was treated as rigid throughout the simulation. We adopted reactive empirical bond order potential function to model the intralayer carbon-carbon interactions within the same $G$ layer while the free $G$ edges were passivated by hydrogen. A registry-dependent interlayer potential that can accurately describe the overall cohesion, corrugation, equilibrium spacing, and compressibility of few-layer G was implemented in the LAMMPS code to model the carbon-carbon interaction between $\mathrm{G}$ flakes. Tersoff potential and Stillinger-Weber potential were utilized for the modeling of $\mathrm{SiO}_{x}$ substrate and silicon (001) layer, respectively. We used a standard 12-6 Lennard-Jones potential for describing $\mathrm{Si}-\mathrm{C}$ and $\mathrm{O}-\mathrm{C}$ long-range vdW interactions according to the Universal Force Field model and the Lorentz-Berthelot mixing rules, whereas $\mathrm{O}-\mathrm{C}$ and $\mathrm{Si}-\mathrm{C}$ covalent bonds at the $\mathrm{G} / \mathrm{SiO}_{x}$ interface were modeled by the Tersoff potential. 
The glue between the tip and the few-layer G nanomesa was simply modeled by applying the Lennard-Jones potential between the silicon layer and the topmost $\mathrm{G}$ layer using a larger $\mathrm{Si}-\mathrm{C}$ interaction energy. The calculations were conducted in the NVT ensemble using the Nosé-Hoover thermostat and Newton's equations of motion were integrated using the velocity Verlet algorithm with a time step of $1 \mathrm{fs}$. The total interfacial force (i.e., $\mathrm{vdW}$ and non-vdW forces) and relative displacement between the innermost G layer and the $\mathrm{SiO}_{x}$ substrate were simultaneously monitored as the tapered silicon (001) layer was pulled in the normal direction with a constant speed of $10^{-2} \AA / \mathrm{ps}$.

\section{Data availability}

The authors declare that the data supporting the findings of this study are available within the paper and its supplementary information files.

Received: 19 December 2019; Accepted: 7 October 2020;

Published online: 05 November 2020

\section{References}

1. Lv, R. et al. Transition metal dichalcogenides and beyond: synthesis, properties, and applications of single- and few-layer nanosheets. Acc. Chem. Res. 48, 56-64 (2015).

2. Rokni, H. \& Lu, W. Nanoscale probing of interaction in atomically thin layered materials. ACS Cent. Sci. 4, 288-297 (2018).

3. Chen, M. K., Rokni, H., Lu, W. \& Liang, X. G. Scaling behavior of nanoimprint and nanoprinting lithography for producing nanostructures of molybdenum disulfide. Microsyst. Nanoeng. 3, 17053 (2017).

4. Chen, M. K. et al. Nanoimprint-assisted shear exfoliation (NASE) for producing multilayer $\mathrm{MoS}_{2}$ structures as field-effect transistor channel arrays. ACS Nano 9, 8773-8785 (2015).

5. Pizzocchero, F. et al. The hot pick-up technique for batch assembly of van der Waals heterostructures. Nat. Commun. 7, 11894 (2016).

6. Britnell, L. et al. Strong light-matter interactions in heterostructures of atomically thin films. Science 340, 1311-1314 (2013).

7. Garcia, J. H., Cummings, A. W. \& Roche, S. Spin hall effect and weak antilocalization in graphene/transition metal dichalcogenide heterostructures. Nano Lett. 17, 5078-5083 (2017)

8. Bediako, D. K. et al. Heterointerface effects in the electrointercalation of van der Waals heterostructures. Nature 558, 425-429 (2018).

9. Velický, M. \& Toth, P. S. From two-dimensional materials to their heterostructures: an electrochemist's perspective. Appl. Mater. Today 8, 68-103 (2017).

10. Khan, M. A. et al. Tunable electron and hole injection enabled by atomically thin tunneling layer for improved contact resistance and dual channel transport in $\mathrm{MoS}_{2} / \mathrm{WSe}_{2}$ van der Waals heterostructure. ACS Appl. Mater. Inter. 10, 23961-23967 (2018).

11. Deng, S. K. \& Berry, V. Wrinkled, rippled and crumpled graphene: an overview of formation mechanism, electronic properties, and applications. Mater. Today 19, 197-212 (2016).

12. Roldán, R. et al. Electronic properties of single-layer and multilayer transition metal dichalcogenides $\mathrm{MX}_{2}(\mathrm{M}=\mathrm{Mo}, \mathrm{W}$ and $\mathrm{X}=\mathrm{S}, \mathrm{Se})$. Ann. Phys. 526, 347-355 (2014).

13. Chen, J., Walther, J. H. \& Koumoutsakos, P. Strain engineering of Kapitza resistance in few-layer graphene. Nano Lett. 14, 819-825 (2014).

14. Wong, J. H., Wu, B. R. \& Lin, M. F. Strain effect on the electronic properties of single layer and bilayer graphene. J. Phys. Chem. C 116, 8271-8277 (2012).

15. Wang, W. et al. Measurement of the cleavage energy of graphite. Nat. Commun. 6, 7853 (2015)

16. $\mathrm{Vu}, \mathrm{C}$. C. et al. Observation of normal-force-independent superlubricity in mesoscopic graphite contacts. Phys. Rev. B 94, 081405 (2016).

17. Koren, E., Lortscher, E., Rawlings, C., Knoll, A. W. \& Duerig, U. Adhesion and friction in mesoscopic graphite contacts. Science 348, 679-683 (2015).

18. Tang, D. M. et al. Nanomechanical cleavage of molybdenum disulphide atomic layers. Nat. Commun. 5, 3631 (2014).

19. Li, B. W. et al. Probing van der Waals interactions at two-dimensional heterointerfaces. Nat. Nanotechnol. 14, 567-572 (2019).

20. Wood, J. D., Harvey, C. M. \& Wang, S. Adhesion toughness of multilayer graphene films. Nat. Commun. 8, 1952 (2017).

21. Koenig, S. P., Boddeti, N. G., Dunn, M. L. \& Bunch, J. S. Ultrastrong adhesion of graphene membranes. Nat. Nanotechnol. 6, 543-546 (2011).

22. Colak, A., Wormeester, H., Zandvliet, H. J. W. \& Poelsema, B. The influence of instrumental parameters on the adhesion force in a flat-on-rough contact geometry. Appl. Surf. Sci. 353, 1285-1290 (2015).

23. Zhao, J. et al. Two-dimensional membrane as elastic shell with proof on the folds revealed by three-dimensional atomic mapping. Nat. Commun. 6, 8935 (2015).

24. $\mathrm{Li}, \mathrm{Z}$. T. et al. Water protects graphitic surface from airborne hydrocarbon contamination. ACS Nano 10, 349-359 (2016).
25. Rooney, A. P. et al. Observing imperfection in atomic interfaces for van der Waals heterostructures. Nano Lett. 17, 5222-5228 (2017).

26. Windom, B. C., Sawyer, W. G. \& Hahn, D. W. A Raman spectroscopic study of $\mathrm{MoS}_{2}$ and $\mathrm{MoO}_{3}$ : applications to tribological systems. Tribol. Lett. 42, 301-310 (2011).

27. Kretinin, A. V. et al. Electronic properties of graphene encapsulated with different two-dimensional atomic crystals. Nano Lett. 14, 3270-3276 (2014).

28. Ryu, Y. et al. Interface-confined doubly anisotropic oxidation of twodimensional MoS 2 . Nano Lett. 17, 7267-7273 (2017).

29. Vasu, K. S. et al. Van der Waals pressure and its effect on trapped interlayer molecules. Nat. Commun. 7, 12168 (2016).

30. Verdaguer, A. et al. Growth and structure of water on $\mathrm{SiO}_{2}$ films on $\mathrm{Si}$ investigated by Kelvin probe microscopy and in situ $\mathrm{x}$-ray spectroscopies. Langmuir 23, 9699-9703 (2007)

31. Uwanno, T., Hattori, Y., Taniguchi, T., Watanabe, K. \& Nagashio, K. Fully dry PMMA transfer of graphene on h-BN using a heating/cooling system. $2 D$ Mater. 2, 041002 (2015)

32. Zhang, X., Jia, F. F., Yang, B. Q. \& Song, S. X. Oxidation of molybdenum disulfide sheet in water under in situ atomic force microscopy observation. $J$. Phys. Chem. C 121, 9938-9943 (2017)

33. Zhang, Y. B., Brar, V. W., Girit, C., Zettl, A. \& Crommie, M. F. Origin of spatial charge inhomogeneity in graphene. Nat. Phys. 5, 722-726 (2009).

34. Yamamoto, M., Einstein, T. L., Fuhrer, M. S. \& Cullen, W. G. Charge inhomogeneity determines oxidative reactivity of graphene on substrates. ACS Nano 6, 8335-8341 (2012).

35. Boukhvalov, D. W. \& Katsnelson, M. I. Enhancement of chemical activity in corrugated graphene. J. Phys. Chem. C 113, 14176-14178 (2009).

36. Elias, D. C. et al. Control of graphene's properties by reversible hydrogenation: evidence for graphane. Science 323, 610-613 (2009).

37. Kumar, S., Parks, D. \& Kamrin, K. Mechanistic origin of the ultrastrong adhesion between graphene and a-SiO 2 : beyond van der Waals. ACS Nano 10, 6552-6562 (2016)

38. Rodrigues, G. D. et al. Strong piezoelectricity in single-layer graphene deposited on $\mathrm{SiO}_{2}$ grating substrates. Nat. Commun. 6, 7572 (2015).

39. Cullen, W. G. et al. High-fidelity conformation of graphene to $\mathrm{SiO}_{2}$ topographic features. Phys. Rev. Lett. 105, 215504 (2010).

40. Seldin, E. J. Stress-strain properties of polycrystalline graphites in tension and compression at room temperature. Carbon 4, 177-191 (1966).

41. Oviedo, J. P. et al. In situ TEM characterization of shear-stress-induced interlayer sliding in the cross section view of molybdenum disulfide. ACS Nano 9, 1543-1551 (2015).

42. Zhu, J. Q. et al. Boundary activated hydrogen evolution reaction on monolayer $\mathrm{MoS}_{2}$. Nat. Commun. 10, 1348 (2019).

43. Li, Z. T. et al. Effect of airborne contaminants on the wettability of supported graphene and graphite. Nat. Mater. 12, 925-931 (2013).

44. Li, X. M., Qiu, H., Liu, X. F., Yin, J. \& Guo, W. L. Wettability of supported monolayer hexagonal boron nitride in air. Adv. Funct. Mater. 27, 1603181 (2017).

45. Kozbial, A., Gong, X., Liu, H. T. \& Li, L. Understanding the intrinsic water wettability of molybdenum disulfide $\left(\mathrm{MoS}_{2}\right)$. Langmuir 31, 8429-8435 (2015).

46. Schrader, M. E. Ultrahigh-vacuum techniques in the measurement of contact angles .5. LEED study of the effect of structure on the wettability of graphite. J. Phys. Chem. 84, 2774-2779 (1980).

47. Amadei, C. A., Lai, C. Y., Heskes, D. \& Chiesa, M. Time dependent wettability of graphite upon ambient exposure: the role of water adsorption. J. Chem. Phys. 141, 084709 (2014).

48. Wei, Y. Y. \& Jia, C. Q. Intrinsic wettability of graphitic carbon. Carbon $\mathbf{8 7}$, 10-17 (2015)

49. Ashraf, A. et al. Spectroscopic investigation of the wettability of multilayer graphene using highly ordered pyrolytic graphite as a model material. Langmuir 30, 12827-12836 (2014).

\section{Acknowledgements}

We acknowledge support from Michigan Center for Materials Characterization (MC) ${ }^{2}$ and Lurie Nanofabrication Facility (LNF) at the University of Michigan. This work was supported in part by the National Science Foundation under Grant No. CMMI-1636132 and in part by the University of Michigan Rackham Predoctoral Fellowship. We also thank Saeedeh Noroozi from Molecular \& Behavioral Neuroscience Institute (MBNI) at the University of Michigan for her assistance with MD simulations.

\section{Author contributions}

H.R. and W.L. conceived the work. H.R. fabricated the devices and performed the experiments. H.R. carried out the finite element and MD simulations and analyzed the data. H.R. and W.L. contributed in the discussion and interpretation of the results. H.R. wrote the manuscript with comments from W.L.

\section{Competing interests}

The authors declare no competing interests. 


\section{Additional information}

Supplementary information is available for this paper at https://doi.org/10.1038/s41467020-19411-7.

Correspondence and requests for materials should be addressed to W.L.

Peer review information Nature Communications thanks Zhuhua Zhang and the other anonymous reviewers for their contribution to the peer review of this work. Peer review reports are available.

Reprints and permission information is available at http://www.nature.com/reprints

Publisher's note Springer Nature remains neutral with regard to jurisdictional claims in published maps and institutional affiliations. (c) (i) Open Access This article is licensed under a Creative Commons Attribution 4.0 International License, which permits use, sharing, adaptation, distribution and reproduction in any medium or format, as long as you give appropriate credit to the original author(s) and the source, provide a link to the Creative Commons license, and indicate if changes were made. The images or other third party material in this article are included in the article's Creative Commons license, unless indicated otherwise in a credit line to the material. If material is not included in the article's Creative Commons license and your intended use is not permitted by statutory regulation or exceeds the permitted use, you will need to obtain permission directly from the copyright holder. To view a copy of this license, visit http://creativecommons.org/ licenses/by/4.0/.

(C) The Author(s) 2020 